\title{
Discrimination of time intervals marked by brief acoustic pulses of various intensities and spectra
}

\author{
PIERRE L. DIVENYI and WILLIAM F. DANNER \\ Central Institute for the Deaf, St. Louis, Missouri 63110
}

\begin{abstract}
Experienced observers were asked to identify, in a four-level 2AFC situation, the longer of two unfilled time intervals, each of which was marked by a pair of 20-msec acoustic pulses. When all the markers were identical, high-level (86-dB SPL) bursts of coherently gated sinusoids or bursts of band-limited Gaussian noise, a change in the spectrum of the markers generally did not affect performance. On the other hand, for $1 \cdot \mathrm{kHz}$ tone-burst markers, intensity decreases below $25 \mathrm{~dB}$ SL were accompanied by sizable deterioration of the discrimination performance, especially at short (25-msec) base intervals. Similarly large changes in performance were observed also when the two tonal markers of each interval were made very dissimilar from each other, either in frequency (frequency difference larger than 1 octave) or in intensity (level of the first marker at least $45 \mathrm{~dB}$ below the level of the second marker). Time-difference thresholds in these two latter cases were found to be nonmonotonically related to the base interval, the minima occurring between 40 - and 80 -msec onset separations.
\end{abstract}

For the past decade, readers of the psychophysics and speech literature have witnessed a growing interest in the perception of temporal aspects of speech (for a review, see Studdert-Kennedy, 1975). Many of the studies generated by this interest deal with the perception of brief time intervals defined by speech sounds. Several phenomena that have been revealed in this area, ones that relate to the perception of voice onset time (VOT), vowel transitions, syllabic duration, etc., are intriguing from the psychoacoustical point of view. Yet, for two reasons, these phenomena, as well as most of the others related to speech perception, do not lend themselves easily to detailed psychophysical analysis. First, the prerequisite for such an analysis would be the definition of what the exact timing cues are in a given speech sound ensemble. Because of the enormous

This research has been supported by a grant from the National Institute for Neurological Diseases and Stroke (NS 03856). The authors wish to acknowledge the assistance they received from James J. Bieker throughout the long months of experimental work, and that of their colleagues at CID for the stimulating discussions that helped to shape the ideas expressed in the article. I. J. Hirsh, D. A. Ronken, and C. S. Watson critically commented on earlier versions of the paper. We wish to thank Professor M. Taibleson and W. J. Kelly for their efforts to scrutinize the mathematical derivation of the model at the first stages of its conception, and W. M. Fisher for making his numerical analysis programs available. At later stages of the mathematical model's development, H. S. Colburn spared no time and effort to point out and help to correct several inaccuracies. P. L. Divenyi's present address: Neurophysiology-Biophysics Laboratories, Veterans Administration Hospital, Martinez, California 94553. W. F. Danner's present address: National Bureau of Standards, Washington, D.C. 20234. Requests for reprints should be addressed to Research Department, Central Institute for the Deaf, 818 South Euclid, St. Louis, Missouri 63110. acoustic complexity of speech, the answer to this question may be very difficult. To appreciate this difficulty, one should remember that phonetic segments differ in their intensity, spectral composition, and duration; in addition, different spectral regions within a given segment may have different buildup and decay characteristics. Indeed, in some instances it may become virtually impossible to determine without ambiguity just when the speech segment in question begins or ends. There is, however, another reason why temporal-perceptual phenomena in speech are difficult to explain in psychophysical terms: the relative paucity of studies dealing with the psychophysics of short time intervals, especially unfilled intervals, that are bounded by simple acoustic (i.e., nonspeech) markers. In our opinion, processes that govern temporal perception of speech and speech sounds cannot be completely understood as long as our knowledge about the processes governing auditory perception of time intervals marked by nonspeech sounds is incomplete. The present study represents an attempt to parametrically investigate the discrimination of brief unfilled time intervals. At the focus of the study is the question of how much time discrimination is influenced by the manipulation of the spectrum and intensity of acoustic markers.

One parameter of particular importance in speech is its spectrum. Our main source of information with respect to spectral effects on time discrimination is the classical study by Henry (1948); it is unfortunate, however, that it had to be conducted without the benefit of the presently available stimulus control. That study showed that discriminability of the duration of pure tones was somewhat better in 
the middle frequency range $(500-2,000 \mathrm{~Hz})$ than at frequencies either below or above this range. Regrettably, no study is known to have dealt with a similar question for unfilled intervals. Furthermore, there is no indication in the literature as to the influence of various noise bandwidths on the discrimination of intervals marked by noise bursts, beyond the finding (Abel, 1972a) that, at equal sound pressure level, the durations of tone bursts and of noise bursts is equally well resolved. For the discrimination of unfilled intervals, not even such a simple tone-vs-noise comparison has been made. Therefore, one must conclude, on the basis of presently available reports, that questions of whether auditory time discrimination is invariant across diverse time-marker spectra or whether there are certain spectral configurations which carry macrotemporal information more effectively than others cannot be unambiguously answered. Yet, temporal discrimination of speech signals almost invariably involves markers that differ in their spectra. Thus, in order to better understand temporal discrimination of speech sounds, one needs to learn about effects of spectral changes on the discriminability of intervals marked by simple acoustic signals, such as tone bursts. The common message of the few available reports on this subject appears to be that, as the frequency difference between two markers increases, detectability of a gap between them (Williams \& Perrott, 1972) or discriminability of a longer $(120-800-\mathrm{msec})$ interval that separates the onsets of the two tones (Divenyi, 1971; van Noorden, 1971) generally worsen. However, these latter experiments have not covered the important temporal range below $100 \mathrm{msec}$. Furthermore, it is not certain whether the detection of a gap and the discrimination of unfilled intervals involve identical mechanisms, for gap detection may well be based on the detection of certain gating transients that are present in the gap-condition and absent in the no-gap-condition.

The other parameter that is of great importance in speech signals and which has been shown to affect temporal discrimination is marker intensity. Several investigators have reported that discrimination of tonal durations becomes more and more difficult as the sensation level of the marker, or its signalto-noise ratio, is decreased (Creelman, 1962; Henry, 1948). The effect seems to be the most pronounced at low marker levels, proving an intuitively obvious point, namely, that the prerequisite for discriminating intervals marked by auditory stimuli is to hear the markers clearly. Unfortunately, intensity effects on the discriminability of unfilled intervals have not been investigated in enough detail, the only study (Abel, 1972b) having explored only relatively highintensity (70-80 dB SPL) noise-burst markers. Thus, while the trend of the intensity effect seems to be as clear as it is predictable, our information, especially about the exact degree of the handicap caused by low-intensity markers, is incomplete.

In the following sections, five experiments are described, experiments which attempted to explore the effects of intensity and spectral content on the discriminability of the duration of unfilled intervals marked by short bursts of tones as well as of bandlimited Gaussian noise. These effects were studied mainly at three base interval durations, each of which has its particular importance in speech: $25 \mathrm{msec}$ (a typical voiced-voiceless CV category boundary), $80 \mathrm{msec}$ (a typical voice onset time [VOT] for English unvoiced-plosive-vowel pairs), and $320 \mathrm{msec}$ (a typical syllable duration). Investigation of temporal discriminability at these three principal intervals was preceded by two studies in our laboratory (Danner, 1975; Divenyi, Danner, \& Bieker, 1974) in which a fine-grain analysis of discrimination inside the 10600-msec range was attempted. These previous experiments demonstrated that the three base durations selected for the present study are a representative set of the whole continuum of durations within that range.

In addition, an attempt is made to integrate the present experimental results into a theoretical framework. Supported by a certain amount of experimental data, Creelman (1962) proposed a simple model for auditory time discrimination, a model which was upheld, with some modification, by Abel (1972a, b). Recently, on the basis of different experimental evidence, Allan and Kristofferson (1974) presented a model different from Creelman's. In a later section of the present paper, we would like to show that Creelman's model describes our data reasonably well and shall propose a somewhat refined version of his theory.

\section{PROCEDURES}

The effect of marker intensity on time discrimination was examined in Experiments 1 and 2, whereas the influence of marker spectrum was investigated in Experiments 3, 4, and 5. In Experiments 1, 2, 3, and 4, the markers were bursts of pure tones; in Experiment 5, the intervals were marked by bursts of band. limited Gaussian noise. The envelope of the bursts was constant throughout the study. It was shaped with a $2.5-\mathrm{msec}$ rise time and a $10-\mathrm{msec}$ fall time, and its total duration was $20 \mathrm{msec}$ (15-msec half-power duration); the contours of the envelope were rounded. This particular envelope shape was chosen in order to emphasize the onset of the markers, since it has been suggested that the offset of the marker is less likely to be a timing cue than its onset (Efron, 1970; Penner, 1975). This envelope contains the energy of the marker burst to a relatively narrow spectral region. The oscillogram of a single tone burst marker is shown in Figure la.

Two pairs of identical tone- or noise-burst markers constituted the stimulus for the experiments. In one of the marker pairs, the onsets of the two bursts were separated by $t$, and in the other pair by $t+\Delta t$ msec. The two time intervals were presented to the subjects in a four-level 2AFC paradigm, that is, in a paradigm where $\Delta t$ could assume, with the same prob- 
ability, any of four preassigned values at any given trial. The first markers of the two intervals were separated by $800 \mathrm{msec}$. A complete trial, including stimulus presentation and response interval, had a total duration of $3.2 \mathrm{sec}$. A diagram of the stimulus is shown in Figure 1b. Stimulus sequencing was accomplished with the help of a set of Tektronix 160-series waveform and pulse generators, a set of Grason-Stadler 1200-series timers and logic modules, and special-purpose digital hardware designed and built by the first author. This device also served to collect data. The tone bursts in Experiments 1-4 were generated by a Wavetek Model 114 triggerable voltage-controlled oscillator; they were all started at a positive-going zero-crossing. The noise bursts used in Experiment 5 were produced by a Grason-Stadler Model 1283 noise generator and filtered by two cascading passive filters (Alison Model 2-BR). To obtain the envelopes described above, the tone or the noise was passed through two electronic switches (Grason-Stadler 1287 and 1287B). In Experiments 1 and 2 , the level of the stimulus was variable, while in all other experiments it corresponded to $86 \mathrm{~dB}$ SPL (about 65-72 dB SL) at the subject's earphone (TDH 49 in MX 41/AR cushions embedded in Rudmose circumaural Otocups). Only the left earphone was active. Temporal parameters of the stimulus were calibrated by means of a Hewlett-Packard Model 5325A electronic counter, with a precision of $.01 \mathrm{msec}$.

Four (three male and one female) extremely capable, paid subjects participated in the experiments. They were selected from a pool of seven subjects who had received several months of previous training in similar time-discrimination experiments. Their ages ranged from 16 to 21 years. Pure-tone audiograms of these subjects showed no anomaly. During the experimental sessions, they were seated in one large soundproof and hypoechoic chamber. They responded by pressing one of two labeled keys ("long-short" and "short-long") to indicate whether, according to their judgments, the longer of the two intervals occurred in the first or in the second position. Visual feedback was given after every response. A typical 1-h session consisted of six blocks of 100 trials. Every datum point reported below is based on $800-1,300$ observations.

The results in subsequent sections will be presented as the time differences ( $\Delta t)$ estimated to be discriminable at a $\mathrm{d}^{\prime}=1.0$ threshold level. These time-discrimination thresholds were obtained by constructing four-point psychometric functions with the data obtained at each of the four time differences used at any given condition. These sets of four points could be fitted with a straight line when plotted as $\mathrm{d}^{\prime}=\mathrm{a}+\mathrm{b} \log \Delta \mathrm{t}$; the fit was generally good to excellent $\left(r^{2} \geqslant .86\right.$ for any condition and for any subject). The time difference at which this straight line intersected the $\mathrm{d}^{\prime}=1.0$ horizontal line was taken as threshold. For each experimental condition, the four time differences were selected such that the resulting four performance levels were distributed, on the average, as evenly as possible within the $55 \%$ to $95 \%$ correct range.

\section{RESULTS}

\section{Experiment 1: Intensity Effects with Identical Markers}

The goal of Experiment 1 was to explore the effect of stimulus level on the discriminability of unfilled time intervals marked by pairs of identical tone bursts. The frequency of the tones was $1 \mathrm{kHz}$ throughout the experiment. Their sensation level was varied from condition to condition within the range of 5-65 dB SL. The sensation level of the tone bursts was established by determining, by the method of limits, their threshold of audibility every time the subject put on his earphones prior to an
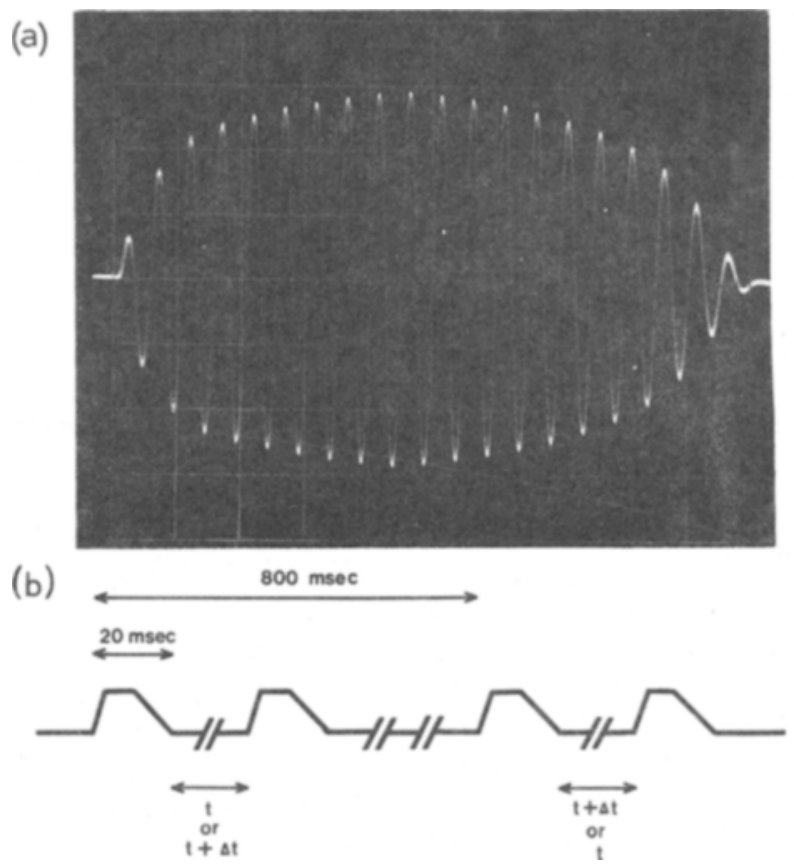

Figure 1. (a) Oscillogram of a single tone burst marker. (b) Schematic representation (half-wave rectified envelope) of the stimulus.

experimental block, to a precision of $\pm 1 \mathrm{~dB}$. Two subjects ( $\mathrm{S} 1$ and $\mathrm{S} 2$ ) participated in the experiment.

Results are plotted in Figures $2 \mathrm{a}$ and $2 \mathrm{~b}$ separately for the two subjects. The figures represent time increments discriminable at threshold as a function of marker level, for the three base durations investigated. The bars indicate \pm 1 standard error of the mean. The broken lines are predictions of the theoretical model described in a later section.

Data of both subjects concur with previous findings (Abel, 1972b; Creelman, 1962; Henry, 1948) concerning the detrimental effect of a reduced marker level on time discrimination. This diminished temporal acuity is most pronounced at the shortest ( $25 \mathrm{msec}$ ) base duration, at which the just discriminable time difference decreased about 10 -fold for one subject (Subject 2) and almost 20-fold for the other (Subject 1), as the stimulus level increased from 5 to $65 \mathrm{~dB}$ SL. It is also apparent in Figure 2 that, as the base intervals became longer, their discriminability was less and less affected by low marker levels. Such a base-duration dependence of the intensity effect is similar to the one found by Creelman (1962).

The most striking, though not altogether unexpected, feature of the present results is that discriminability of short unfilled intervals does not deteriorate by more than a factor of 2 , even with a $40-\mathrm{dB}$ attenuation of the markers. Indeed, for the 25 -msec base interval, the subjects do not seem to have difficulty discriminating even very small time differences as long as the level of the marker 


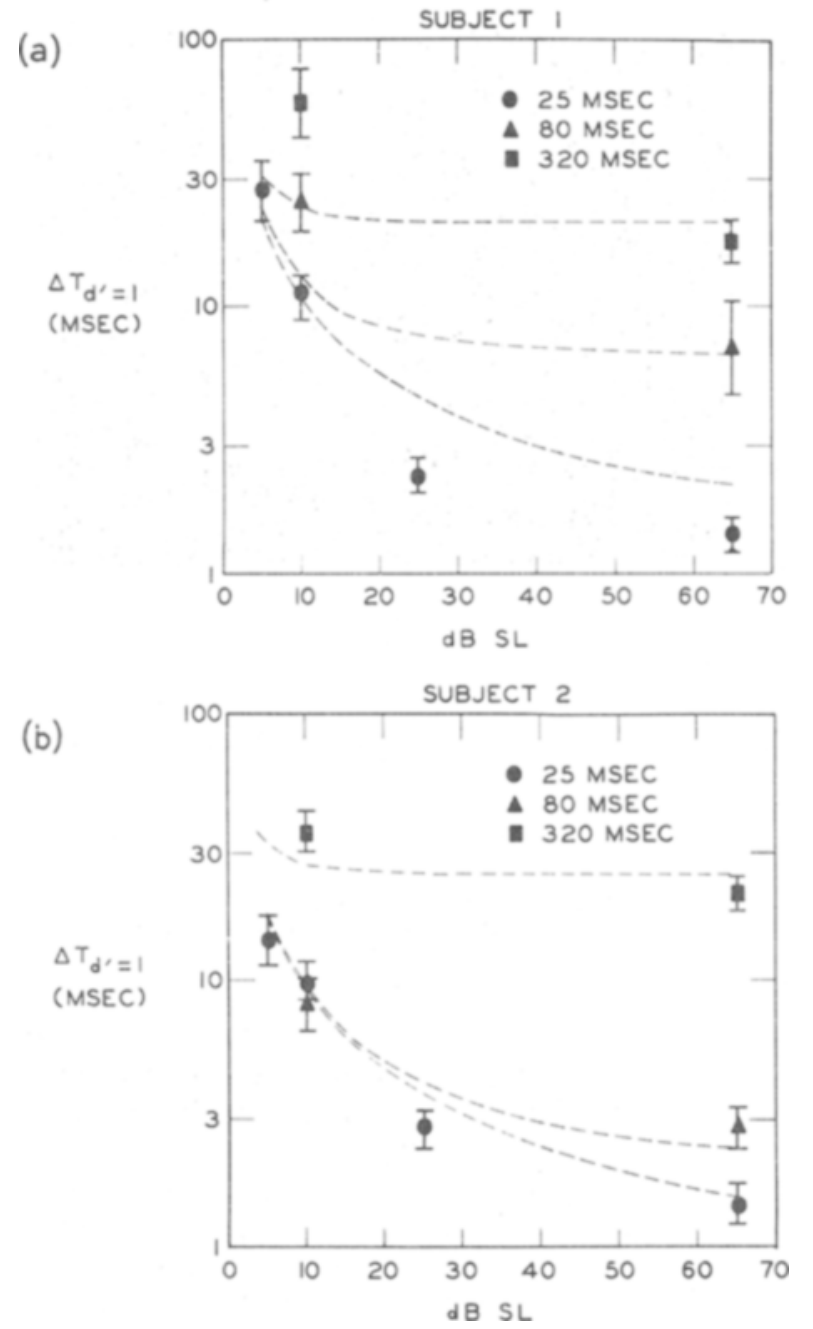

Figure 2. Results of Experiment 1. On the abscissa: sensation level of each tone burst marker in the stimulus. On the ordinate: time difference discriminable at the $d^{\prime}=1.0$ level. Parameter: base interval duration. The bars indicate \pm 1 standard error of the mean. The broken lines refer to time difference thresholds predicted by the model. Panel a, Subject 1; panel b, Subject 2.

is at least $25 \mathrm{~dB}$ SL; almost all the effect takes place in the 20 -dB range between 25 and $5 \mathrm{~dB}$ SL. The reader should note that at $5 \mathrm{~dB}$ SL the signals are still audible, albeit faint.

\section{Experiment 2: Intensity Effects with Markers Presented at Different Levels}

In the former experiment, all markers had the same intensity. In speech, however, intensities of contiguous segments are only seldom equal. Thus, Experiment 2 was an attempt to examine changes in the discriminability of time intervals that were induced by decreasing the level of the first marker in each of the two intervals that the subjects had to compare. In the stimulus of this experiment, the intensity of the second and fourth marker shown in Figure $1 \mathrm{~b}$ was held constant at $86 \mathrm{~dB}$ SPL, while that of the first and the third marker was simul- taneously decreased by a given number of decibels. As in Experiment 1, all markers were $1-\mathrm{kHz}$ tone bursts. The same two subjects participated in the experiment.

Results for the two subjects are shown separately in Figures 3a and 3b. Large intensity differences between the two markers of each interval appear to have considerably interfered with the subjects' task to decide which of the two intervals was longer. On the other hand, moderate attenuation of the first marker (up to $25 \mathrm{~dB}$ for Subject 1 and up to $45 \mathrm{~dB}$ for Subject 2) had only a relatively small effect. As in
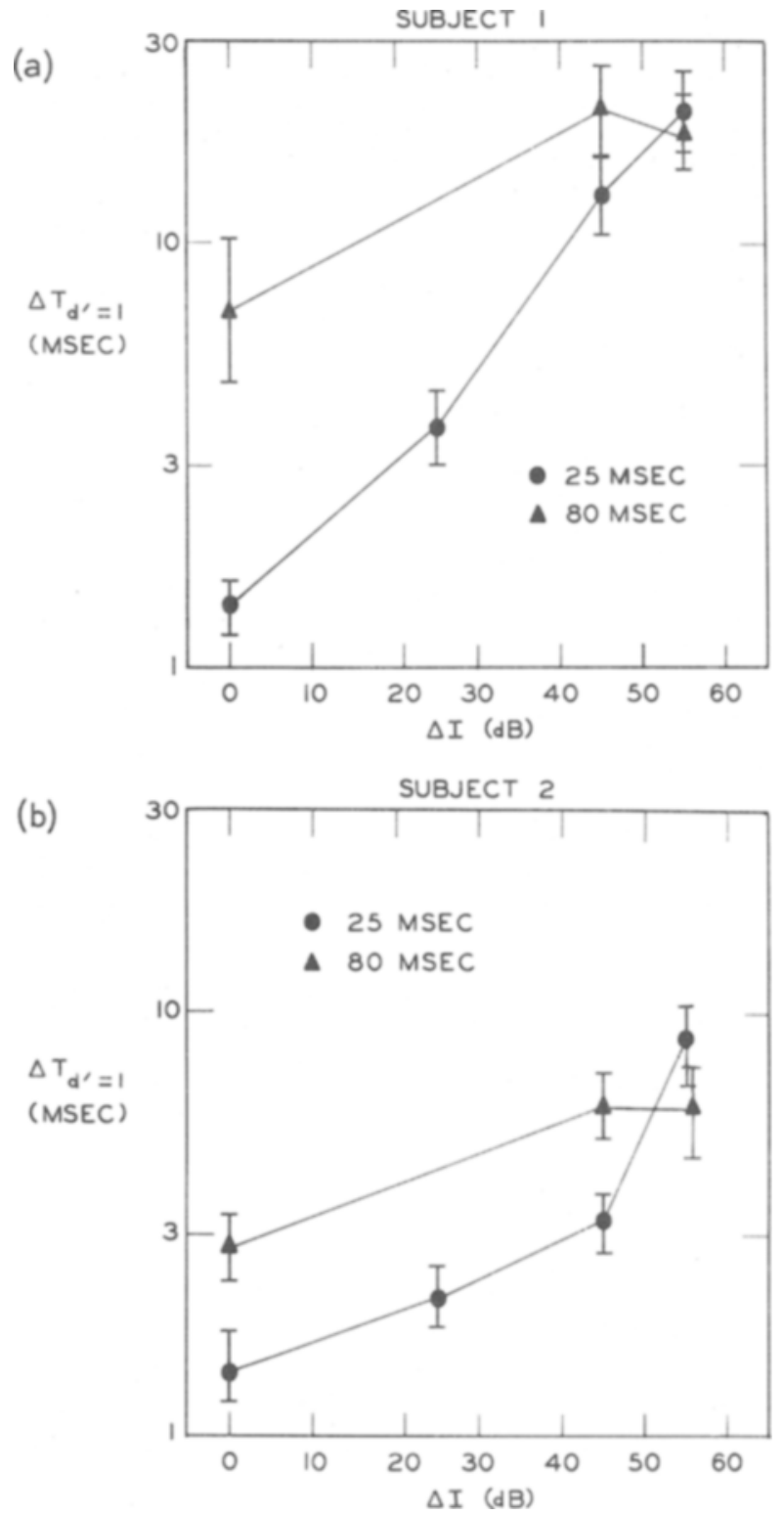

Figure 3. Results of Experiment 2. On the abscissa: the difference $(\Delta I)$ between the intensity of the second marker of each interval (86 $\mathrm{dB}$ SPL) and the intensity of the first marker of each interval. On the ordinate: time difference discriminable at the $d^{\prime}=1.0$ level. Parameter: base interval duration. The bars refer to \pm 1 standard error of the mean. Panel a, Subject 1; panel b, Subject 2. 
Table 1

Backward-Masked Thresholds (Decibels SPL) for a 20-Msec 1-kHz Tone Burst Followed by a 20-Msec 1-kHz 86-dB SPL Tone Burst

Temporal Separation (Onset to Onset) in Milliseconds

\begin{tabular}{llllllr} 
& 25 & 30 & 38 & 50 & 80 & 100 \\
\hline Subject 1 & 35 & 33.5 & 30 & 20 & 13.5 & 15 \\
Subject 2 & 26.5 & 21.5 & 25 & 17.5 & 13.5 & 15 \\
\hline
\end{tabular}

the former experiment, the attenuation interferes with temporal discrimination more at short $(25-\mathrm{msec})$ base durations than at longer ones, a point which suggests that some type of backward masking was responsible for the observed performance decrement. For this reason, backward-masked thresholds of audibility were determined for the first marker followed by the 86-dB SPL second marker, at several temporal separations. These thresholds are summarized in Table 1. Comparison of these audibility thresholds with the time-increment discrimination thresholds shown in Figure 3 reveals that, with only minor exceptions, time discrimination performance became poor only when the level of the first marker was near its threshold of audibility (barely above or, sometimes, below). In these cases, the subject could hear only one marker in the "short" interval, i.e., the one in which the markers were separated by $\mathrm{t}$ msec. Thus, in order for him to distinguish such intervals from intervals having a $t+\Delta t$ msec separation between the two markers, $\Delta t$ had to be sufficiently long, so that he could hear two markers. In other words, time discrimination became an apparently less efficient one-sound-two-sound discrimination, thereby requiring rather large just-noticeable time differences.

Another feature of interest appears in Figure 3. Comparing just-noticeable time differences obtained at the 25 - and $80-\mathrm{msec}$ base durations in the large- $\Delta \mathrm{I}$ conditions, one notices that the two $\Delta t$ values overlap. Because of this interesting phenomenon, we examined in some detail the base duration continuum for these large intensity differences. Results of this extension of Experiment 2 are shown in Figure 4 for Subject 1 , at a $\Delta \mathrm{I}$ of $45 \mathrm{~dB}$, and for Subject 2, at a $\Delta \mathrm{I}$ of $55 \mathrm{~dB}$. It is not without some surprise that a nonmonotonicity in the time-difference discrimination function is noted: there is a time difference threshold minimum at a base duration lying in the middle of the presently investigated range. Whether this intermediate base duration (45 msec for Subject 1 and $80 \mathrm{msec}$ for Subject 2) is truly the one at which time discrimination is best for the given subject and the given attenuation could be decided only after systematic scanning of the whole range. Until such a systematic exploration is accomplished, one must be satisfied with the observation that time difference thresholds are nonmonotonically related to base duration whenever the second marker's backward-masking influence severely decreases the first marker's sensation level. Similar nonmonotonicity has been hitherto observed only with speech (Liberman, Harris, Kinney, \& Lane, 1961 ) or speech-like (Miller, Pastore, Wier, Kelly, \& Dooling, 1976) stimuli.

\section{Experiment 3: Spectral Effects with Identical Tone-Burst Markers}

Experiment 3 was to investigate whether the frequency of a pair of tone bursts affected the discriminability of the interval enclosed by them. Three base intervals, 25,80 , and $320 \mathrm{msec}$ (from marker onset to marker onset), were tested in the experiment at three frequencies, $.5,1$, and $4 \mathbf{k H z}$. All tone bursts were gated at a positive-going zero crossing. The stimulus level was held constant at $86 \mathrm{~dB}$ SPL.

Combined results of three subjects (Subjects 2, 3, and 4) are shown in Figure 5, in which, as in the

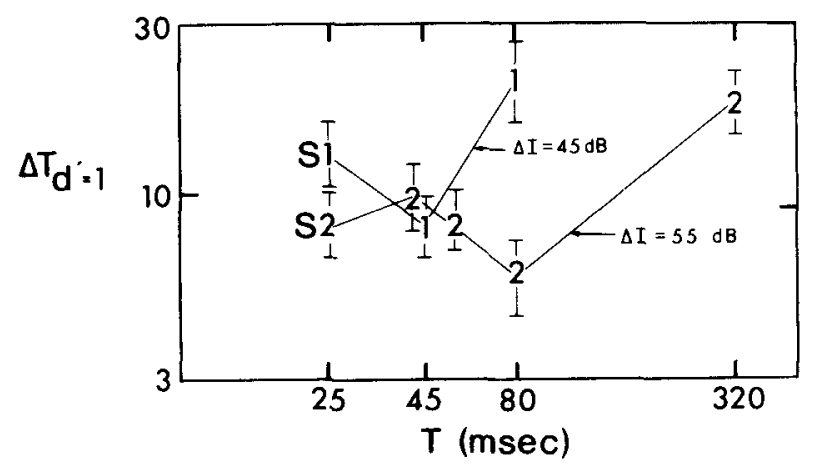

Figure 4. Results of Experiment 2. On the abscissa: base interval duration. Ordinate as above. Parameter: Subject 1 at $\Delta I=45 \mathrm{~dB}$; Subject 2 at $\Delta I=55 \mathrm{~dB}$.

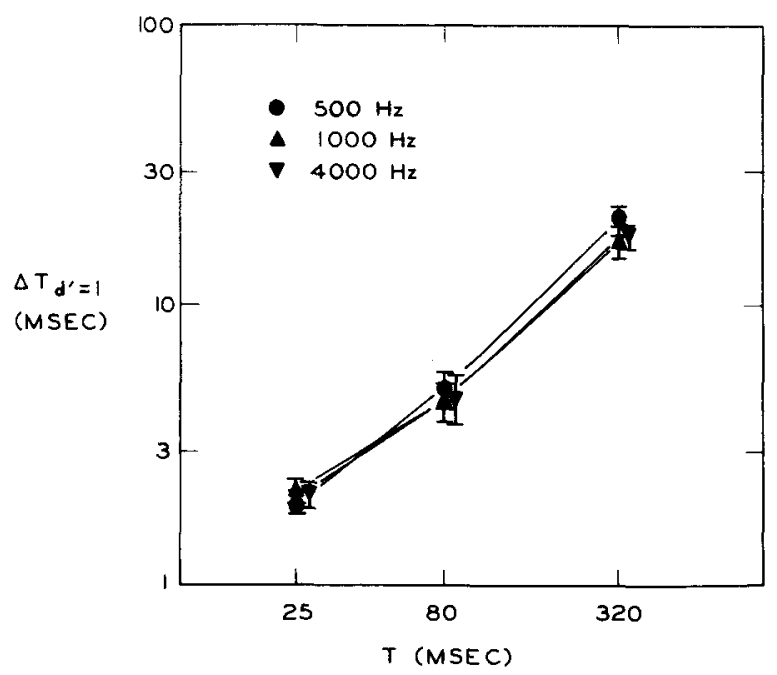

Figure 5. Results of Experiment 3. On the abscissa: base interval duration. Ordinate: time difference discriminable at threshold. Parameter: marker frequency. Average data of three subjects. Bars represent \pm 1 standard error of the mean computed for the three subjects' pooled data. 
previous figure, time differences discriminable at threshold are represented as a function of base duration for the three marker frequencies. These results clearly suggest that, at least for the base intervals examined, the frequency of short tones had no influence whatsoever on the discriminability of an interval defined by two such tones.

\section{Experiment 4: Spectral Effects with Tone Burst Markers Presented at Different Frequencies}

Because temporally contiguous speech segments are, with almost no exception, different from each other in their spectral composition, broad interpretation of the lack of spectral effects found in Experiment 3 would be risky. In order to have only a hint of the role played by spectral attributes in temporal discrimination of speech, one must address the question of whether spectral changes in the markers can cause possible changes in the discriminability of a given unfilled interval when the spectra of its two marker sounds are made more and more different from each other. Experiment 4 was conducted to explore this question in its simplest form, that is, with markers consisting of sinusoids different in frequency. One frequency only was used for the first markers of the two intervals to be compared, whereas the second markers of the two intervals had another, different frequency. The geometric mean of the two frequencies was held constant at $1 \mathrm{kHz}$ throughout the experiment. Five frequency differences were explored: $0,1 / 2$ octave $(358 \mathrm{~Hz}), 1$ octave $(714 \mathrm{~Hz}), 2$ octaves $(1.5 \mathrm{kHz})$, and 4 octaves $(3,750 \mathrm{kHz})$. The frequency change in the stimulus was always descending, that is, the first marker of each interval had a higher frequency than the second marker. The stimulus level corresponded to $86 \mathrm{~dB}$ SPL at the subject's earphone. Two subjects (Subjects 1 and 2) participated in the experiment.

Figure 6 shows the results for a 25 -msec base interval. It is evident that discriminability of a given time difference is best when both markers have the same frequency. As the two markers are made more and more dissimilar from each other in frequency, time discrimination performance gradually deteriorates. For Subject 1, this deterioration is continuous along the 4-octave range tested, whereas it stops at the 2-octave difference for Subject 2. The amount of performance decrement is relatively large: about fourfold for both subjects at those frequency differences at which they require the largest just-noticeable time differences. These data are concordant with those found for much larger base intervals in other time interval discrimination studies (Collyer, 1974; Divenyi, 1971; van Noorden, 1971); they are also in agreement with results on the detectability of a temporal gap between two tones different in frequency (Williams \& Perrott, 1972).

With the frequency difference held constant at 2 octaves, base intervals longer than $25 \mathrm{msec}$ were

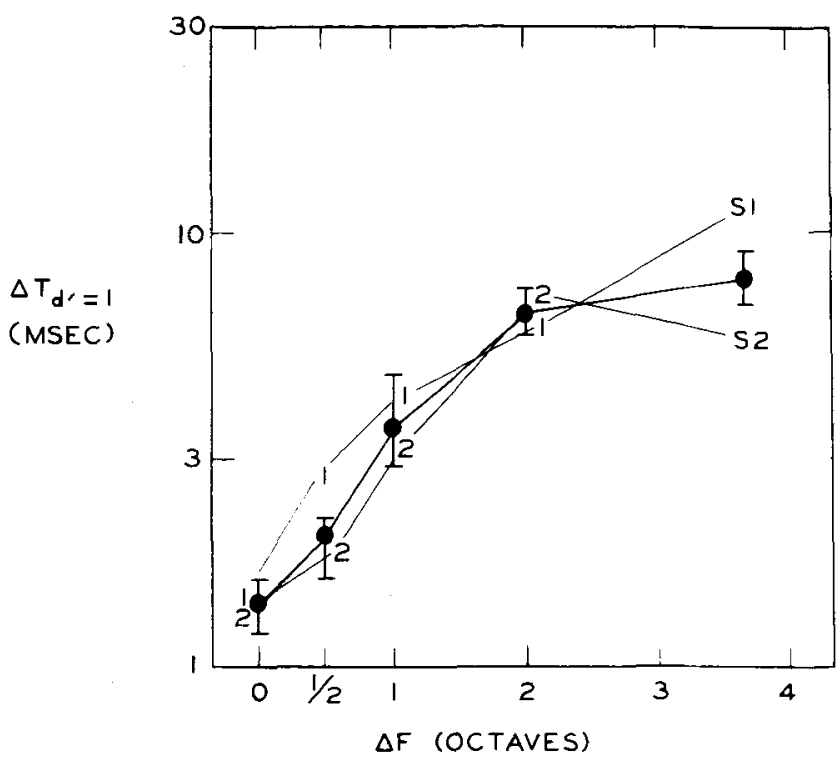

Figure 6. Results of Experiment 4 for a base duration of 25 msec. Abscissa: the difference between the frequency of the first marker and the frequency of the second marker in octaves. The geometric mean of the two frequencies is constant at $1 \mathrm{kHz}$. Ordinate: time difference discriminable at threshold. Data of two subjects.

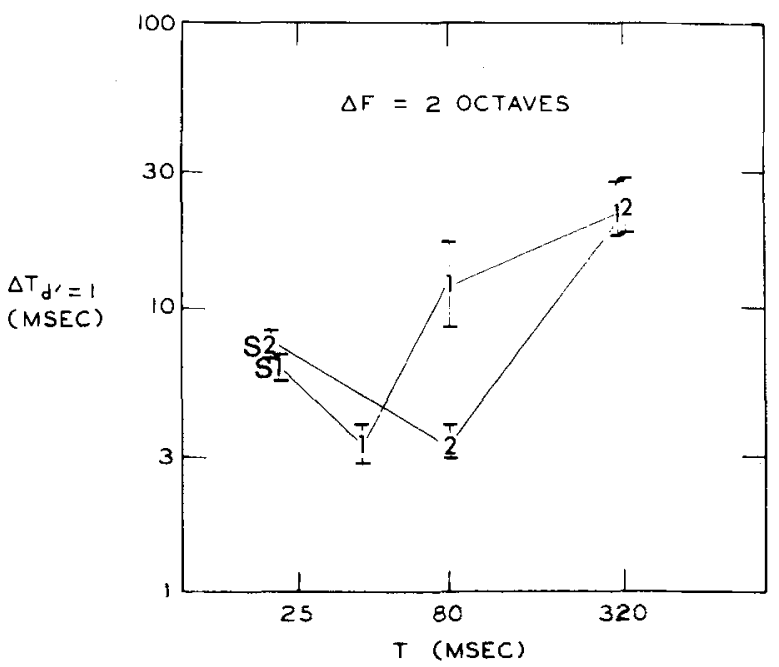

Figure 7. Results of Experiment 4 for a 2-octave frequency difference. Abscissa: base interval duration. Ordinate: time difference discriminable at threshold. Data of two subjects.

also tested. Time discrimination thresholds are shown in Figure 7 as a function of base interval for the two subjects.

As was seen for the case of time markers different in intensity (Figure 4), these results suggest that brief time markers different in frequency will also give rise to nonmonotonic time discrimination functions similar to those found in VOT discrimination (Liberman et al., 1961). 


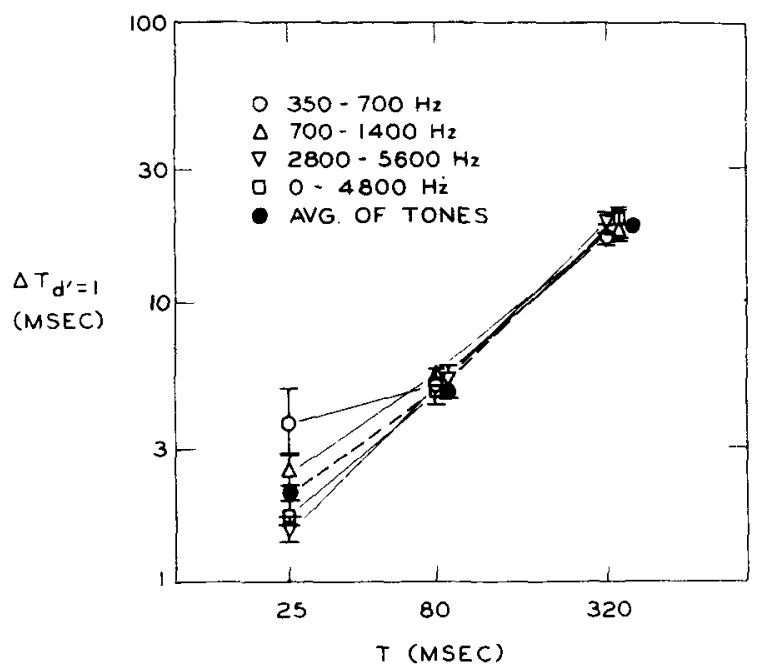

Figure 8. Results of Experiment 5. Abscissa: base interval duration. Ordinate: time difference discriminable at threshold. Parameter: center frequency of the noise marker. Data of Experiment 3, averaged across the three tone frequencies, are also shown. A verage results of three subjects.

\section{Experiment 5: Spectral Effects with Identical Noise Burst Markers}

The conclusion of Experiment 3 was that, when the frequency of tone burst markers is the same within a stimulus sequence, the subjects' accuracy to discriminate short time intervals between such markers is independent of the frequency of the tone bursts. The objective of Experiment 5 was to see whether this conclusion could be extended to the case where the intervals are marked by filtered noise bursts instead of tone bursts. The noise, shaped to yield the amplitude characteristics of the tone burst markers used in the previous experiments (Figure 1a), came from a Gaussian source whose bandwidth was limited to 1 octave prior to going through the electronic switches. The center frequency of the octave-band noise was $.5,1$, or $4 \mathrm{kHz}$. In addition to these three bandpass conditions, a broad-band low-pass condition $(0-4,800 \mathrm{~Hz})$ was also included. Regardless of the noise bandwidth, the total average noise power was fixed at $86 \mathrm{~dB}$ SPL at the subject's earphone. Three subjects (S2, S3, and S4) participated in the experiment.

Time difference thresholds for the various noise marker conditions are shown as a function of base duration in Figure 8 for data of the three subjects combined. To provide a basis for comparison of the present results with those obtained for tone bursts, the thresholds found for the same subjects in Experiment 3, averaged across the three tone-burst frequencies used, are also displayed in the same figure.

Like previous findings for the discriminability of the duration of tone bursts and noise bursts (Abel, 1972a), these results suggest that discriminability of unfilled intervals does not become different when noise burst markers are substituted for tone burst markers. Also, changing the spectrum of the noise marker seems to have little effect on time discrimination performance. There is only one case in which some effect may be present: discriminability of the shortest $(25-\mathrm{msec})$ interval suffers as the center frequency of the noise becomes lower and lower. This effect, though significant, is nonetheless slight: time discrimination thresholds obtained for the noise markers centered at $500 \mathrm{~Hz}$ are little over twice the size of the thresholds obtained for the high-center frequency noise, the wide-band noise, or the tone burst markers. There is, however, a confounding factor which makes the interpretation of this small effect difficult. Since it is the logarithmic, rather than the absolute, bandwidth that was held constant in the bandpass conditions, a decrease in the center frequency of the octave-band noise amounted to a decrease in its absolute bandwidth. Thus, the above results fail to distinguish center frequency- and bandwidth-related effects. In order to make such a distinction possible, discriminability of the $25-\mathrm{msec}$ base interval was examined in more detail using several additional bandpass conditions. The bandwidth of the noise was either $350 \mathrm{~Hz}$ or $2.8 \mathrm{kHz}$; its center frequency $(\log )$ was $.5,1$, or $4 \mathrm{kHz}$. Again, the total average noise power was constant at $86 \mathrm{~dB}$ SPL.

These results are shown in Figure 9 for the average data of two subjects (Subjects 2 and 3). There is a strong suggestion that it is the absolute bandwidth rather than the spectral location of the noise which is the determinant factor in influencing time discrimination at such short base intervals. Noise bursts having a narrower bandwidth appear to mark time

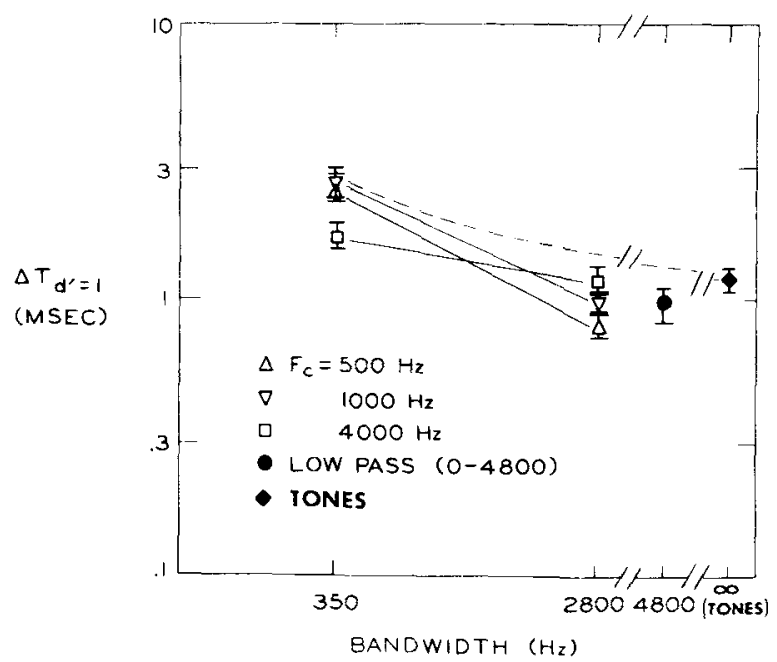

Figure 9. Results of Experiment 5 for a 25-msec base interval. Abscissa: noise marker bandwidth in hertz. Ordinate: time difference discriminable at threshold. Parameter: noise center frequency. The broken line indicates theoretical results as predicted by the model. Two subjects' average data. 
somewhat less efficiently than do noise bursts whose bandwidth is large. This conclusion may be somewhat estopped by the lack of difference at the wide and narrow noise bands centered at $4 \mathrm{kHz}$; however, this particular lack of effect is possibly apparatusrelated.'

While the size of the bandwidth effect is moderate at most (less than \pm 2 standard errors of the mean), it may be nonetheless important because it suggests that the efficiency of an auditory signal to mark time is inversely related to the extent of short-term fluctuations in its power. Such fluctuations are absent in coherently gated tones and are negligible in wideband noises, but become more and more noticeable as the bandwidth of the noise becomes narrower and narrower.

\section{DISCUSSION}

The above experiments examined the dependence of human sensitivity to differences in the duration of unfilled auditory intervals on the intensity and the spectrum of the time-marker acoustic signals. From the perspective of these experiments, auditory discrimination of unfilled intervals appears to be only moderately dependent on these two classes of marker parameters (calling "moderate" any effect smaller than \pm 2 standard errors of the mean), provided that two conditions are fulfilled: (1) the two signals that mark the interval are clearly audible, and (2) the two signals are not overly dissimilar in their spectrum. Discriminability of a time interval has been seen to suffer to a great extent only when either of these two conditions was severely violated. To violate the first condition, either one or both of the two markers had to be presented at a level not higher than $10 \mathrm{~dB}$ above their threshold of audibility; to violate the second condition, the frequency of one marker had to be presented at least 1 octave away from the frequency of the other marker. Moreover, impairment of temporal discrimination performance, when one of these severe violations occurred, was found to be sizable only when the intervals to be discriminated were short $(25 \mathrm{msec}$ between marker onsets). Thus, for longer unfilled intervals and for all intervals marked by pairs of clearly audible signals identical or nearly identical in their spectral composition, temporal discrimination is generally uniform across a wide range of intensities and spectra.

Two general conclusions stem from the results. The first is that, in auditory perception of time, the ear's role seems to be limited to simply signaling to some higher up center the occurrence of a change in the stationarity of the acoustic environment, that is, the occurrence of an acoustic event. The second conclusion is that the auditory system appears to be a somewhat inefficient integrator of macrotemporal information over the spectrum. In terms of auditory information processing, these conclusions suggest that every auditory channel, or a combination thereof, could be used by the nervous system with equal effectiveness for timing external events, as long as the level of the signals that reach these channels is high enough to leave little uncertainty as to the moment of their occurrence. However, in order for these moments of occurrence to be efficiently transmitted to the centers responsible for time perception, there seems to be a requirement that successive auditory events be processed by the same channel or channels. Thus, having trodden paths different from those of Bregman and Campbell (1971), we arrived at a similar conclusion, namely, that sequential auditory information is broken up by the perceptual system into separate streams which are segregated on the basis of their spectral coherence, and that reception of temporal information across streams is severely degraded. Auditory stream segregation and influence of frequency differences on auditory time discrimination both point toward a certain spatial dependence of auditory perception of time, in the neuroanatomical sense. Such a spatial dependence would mean that, despite the apparent accuracy of the nervous system to resolve short auditory intervals, the auditory stimulus must undergo a great deal of preprocessing before it is able to signal the occurrence of an acoustic event to a more central timing mechanism. It is interesting to note that, although such a (probably multistage) processing would have to impose a delay as well as a transmissionjitter on the trace of the time marker, the auditory system is still the most efficient transmitter of temporal information among all sensory modalities (Hirsh \& Sherrick, 1961).

One is tempted to attribute this spatial dependence of time perception to some type of "filtering," one which may be similar to the filtering assumedly taking place when off-target frequencies are ignored by the listener (Greenberg \& Larkin, 1968). According to such a view, the above-observed increasingly deteriorating temporal discrimination with increasing marker frequency differences could be the result of the inability of the listener to effectively attend to the onset of an auditory signal whose energy is concentrated outside a currently active listening band. This is to say that, for the purpose of temporal information transmission at least, the otherwise loud signals may have become "attenuated" to an extent proportional to the frequency difference between the previous and the present marker signal. Therefore, one may think that the frequency-difference effect seen in Experiment 4 and the intensity effect seen in Experiment 1 would have a common origin, namely, attenuation of temporal information. An actual mapping of the time difference thresholds obtained in Experiment 4 (Figure 7) onto those obtained in Experiment 1 (Figure 2) produces the 


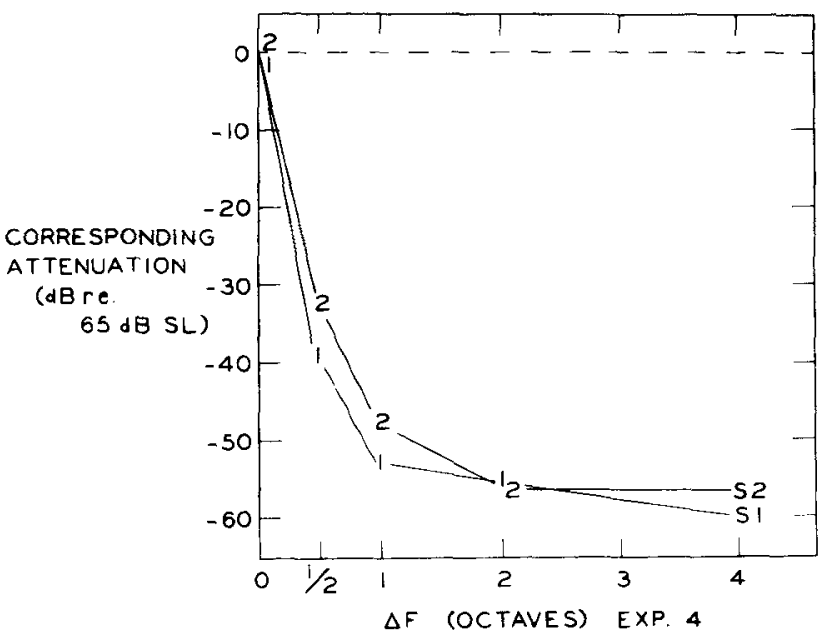

Figure 10. Attentional filter profile for Subjects 1 and 2. Abscissa: frequency difference between the two markers. Ordinate: marker intensity at which the time difference threshold was found to be identical in Experiment 1 (Figure 2) to the one obtained in Experiment 4 for the particular frequency difference (Figure 6).

"attentional filter profiles" shown for Subject 1 and Subject 2 in Figure 10. Such a filter is not without resemblance to the one proposed by Treisman (1960) in her attenuation theory of attention, although whether the two filters are actually equivalent or not would be difficult to decide. However, the representation in Figure 10 permits one to establish the slope of this peculiar auditory filter, one which leaves sensation level intact but which is spectrally selective toward temporal information carried by the auditory signals. The profiles computed for Subjects 1 and 2 almost completely overlap; yet their time discrimination performance in both Experiments 1 and 4 displayed some important differences. According to this figure, the response of the filter falls off at a rate of about $60 \mathrm{~dB} /$ octave up to a frequency difference of 1 octave and remains basically flat thereafter. $^{2}$

Beside these major conclusions, the results also suggest another point. This point concerns the physical attribute of an auditory signal that is most likely to be responsible for time marking. In Experiment 5, we saw that discrimination of short time intervals was influenced by the extent of fluctuations in the power of noise burst markers. Therefore, it seems that when the auditory system is employed for the sole purpose of registering the onset of signals, it acts like an energy detector. More will be said about this subject in the next section, where a modified version of Creelman's (1962) model will be described.

It is of some interest to compare the present data with those found in other time discrimination studies. Figure 11 shows discrimination functions (time difference threshold plotted against base interval dura- tion) obtained in the present experiments (average data for tone- and noise-burst markers), as well as data reported by Chistovich (1959) for the discrimination of intervals bounded by clicks, by Abel $(1972 b)^{3}$ for the discrimination of intervals bounded by 5 -msec broad-band noise bursts, and by Creelman (1962) for the discrimination of the duration of $1-\mathrm{kHz}$ tone bursts in noise. All these $\log \Delta t-v s-\log t$ functions may be reasonably well fitted by straight lines. There are, however, important differences between the present data and data of the other three workers. First, the slope of our data is almost unity (.93), whereas those calculated for the other three sets of results are smaller $(.74, .77$, and .78 , respectively). Therefore, our results come close to supporting the view that Weber's law holds for time discrimination, thus concurring with recent findings by Getty (1975). Also, more importantly, our data represent better discrimination performances than those reported in the other studies. The average $\Delta t / t$ value computed for the three base durations of the present experiments is only .063 as compared to the average $\Delta t / t$ fraction computed for the three other sets of data combined, which is almost exactly $.2{ }^{4}$ The reason for such a great discrepancy may lie in the fact that our observers went through a training period of several months, during which their performance (fairly good already at the beginning) constantly improved. Other time discrimination studies, with the exception of that of Creelman (1962), did not report using similarly long training periods. The data presented in this paper, therefore, may well approximate the ultimate limits of what a human observer can do in a situation where the task is to discriminate short unfilled auditory intervals.

Finally, the nonmonotonic time difference func-

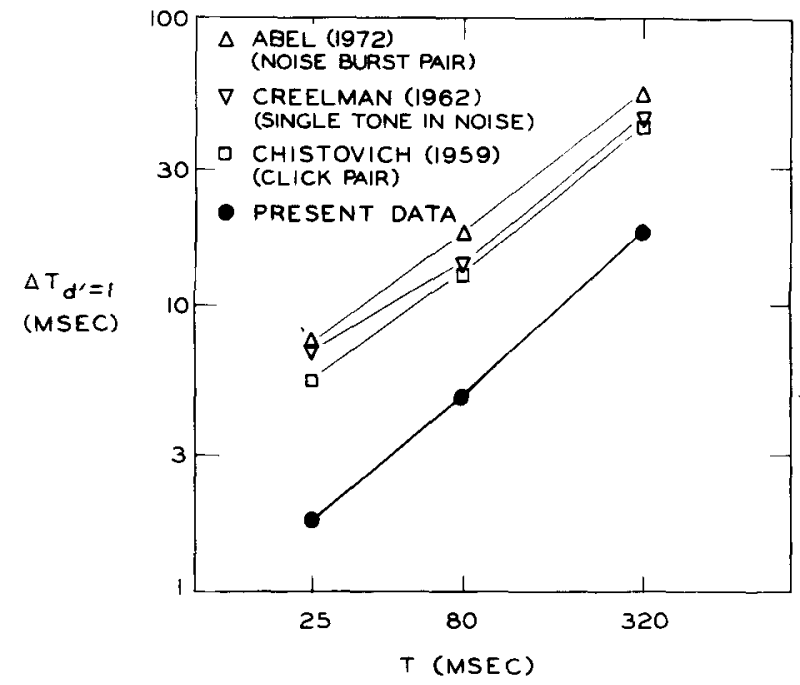

Figure 11. Comparison of the present data (average results of Experiment 3) and data reported by other workers: Chistovich (1959), Abel (1972b), and Creelman (1962). Abscissa: base interval duration. Ordinate: time difference discriminable at threshold. 
tions obtained in Experiments 2 (variable-intensity markers) and 4 (variable-frequency markers) show some resemblance to nonmonotonic time discrimination functions obtained in VOT discrimination experiments (Abramson \& Lisker, 1970; Liberman et al., 1961), as well as those obtained for the discrimination of the interval separating a noise and a buzz (Miller et al., 1976). Since the present tone burst pairs of different intensities and frequencies cannot be classified as speech or speech-like sounds, one has to conclude that the nonmonotonic behavior of time difference discrimination functions is not a privileged attribute of speech. This conclusion is only somewhat weakened by the fact that the threshold minima found in the two experiments in question (Figures 4 and 7) are not in agreement for the two subjects, on the one hand, and that these minima are located at base interval durations considerably longer than the 20-35-msec VOT intervals at which such minima have been observed when speech stimuli were used. The discrepancies could well be experiencerelated, since, if the nonmonotonicity in the speech discrimination functions is truly related to experience as suggested by several authors (Abramson $\&$ Lisker, 1970; Liberman et al., 1961), the 12-odd months during which our subjects were exposed to these stimuli represent a training period that is far shorter than the decades of experience the human has with speech sounds.

The psychophysicist is likely to attribute the nonmonotonicity seen in the present data to two forces exerting opposite effects: to some psychophysical law (e.g., Weber's law), which should account for the degrading performance as the base intervals become longer and longer, and to temporal masking (Figure 4) or auditory streaming (Figure 7), which gains more and more importance as the temporal separation between the two markers becomes shorter and shorter. Thus, if similar processes were to operate in speech perception, our results would support the hypothesis advanced by Miller et al. (1976), who argued that the decrement in VOT discrimination performance customarily found at short VOTs is attributable to temporal masking. If this were the case, the similarity between the present nonspeech time discrimination data and the VOT discrimination data reported by these other authors could have only two possible explanations. The first of these is that intensity disparity or spectral disparity between two simple acoustic markers may make them more "speech-like." The second explanation is that the processes involved in certain nonspeech discrimination tasks may not be too remote from those that intervene in speech perception. Recent findings on voiced-voiceless CV-pair identification by chinchillas (Kuhl \& Miller, 1975), as well as on VOT discrimination of filtered pairs of CV-like synthetic sounds by humans (Divenyi \& Danner, 1975), appear to support the second of these two hypotheses. A more detailed scrutiny of VOT discrimination will be the topic of a subsequent report.

\section{IMPLICATIONS FOR A MODEL OF TIME DISCRIMINATION: CREELMAN REVISITED}

The dimension of time is elusive and, whether looked upon from a philosophical, scientific, or artistic point of view, most complex as a concept (Fraser, 1966). It should be, therefore, no surprise to anyone that psychophysics of time has some inherent, particular problems. Possibly it is due to these difficulties that, as we mentioned earlier, temporal psychophysics has been a relatively neglected branch of the field of perception. This complexity may also explain the fact that most experimental reports on time discrimination are accompanied by, or at least make extensive reference to, some particular theoretical model.

By necessity, most psychophysical theories represent oversimplified reflections of what one believes to be the actual perceptual-neurological processes that they try to portray; theories treating the psychophysics of time are guilty of the same oversimplification and are to be considered only more or less successful approximations. Theoretical models of time discrimination fall into two main categories: (a) counter models, the most representative of which is that of Creelman (1962), and (b) quantal models, a clear example of which has been described by Allan and Kristofferson (1974). The major difference between the two models is that, whereas the counter model predicts the just discriminable time difference, $\Delta t_{\text {threshold, to be mono- }}$ tonically related to the base interval, $t$, these two quantities are predicted to be independent by the quantal model. The quantal model, both in its original and in its more advanced two-stage version ("onset-off set model," Allan \& Kristofferson, 1974), has received support from experimental results obtained mainly by Kristofferson and his associates. On the other hand, a large number of authors, working in various laboratories (e.g., Abel, 1972a, b; Chistovich, 1959; Creelman, 1962; Divenyi, 1971; Getty, 1975), have presented concordant evidence of a monotonic relation between $\Delta t_{\text {threshold }}$ and $t$, thereby lending support to the counter model. Also, the present data (see Figures 5, 8, and 11), as well as other data collected earlier in our laboratory (Danner, 1975; Divenyi et al., 1974), fall unambiguously in this second category. This is why it was the counter model, rather than the quantal model, that we attempted to apply to the present results. We did this by bringing two modifications to Creelman's (1962) original model, one for practical and one for conceptual reasons.

The elegant theory that Creelman (1962) proposed to model auditory time discrimination can be de- 
composed into two separate stages: a presumably central timer stage and an independent, presumably sensory, turn-on-turn-off stage.

The timer stage. To represent the internal timer, the model postulates a simple counter which is incremented by every discharge emitted by a random, neural pulse source for the duration of the time interval under observation. Physical durations are discriminated by the subject on the basis of a comparison of the number of counts accumulated during each interval: the larger the number of counts, the longer the interval will be judged. The pulse source is assumed to obey the Poisson law with a constant average pulse rate (called intensity), of $\lambda$ counts $/ \mathrm{sec}$. Since the number of counts accumulated during any given interval is expected to be large, its distribution will approach normality. Thus, by definition, discriminability of two intervals of durations $t$ and $\mathrm{t}+\Delta \mathrm{t} \mathrm{sec}$, respectively, are expressed in $\mathrm{d}^{\prime}$ units as 2 times the normalized difference between the means of the two Poisson distributions, i.e., the difference between the two means divided by the square root of the sum of the variances, or

$$
\mathrm{d}^{\prime}{ }_{2 \mathrm{AFC}}=\frac{\sqrt{2}\left(\mu_{\mathrm{t}}+\Delta \mathrm{t}-\mu_{\mathrm{t}}\right)}{\left(\sigma_{\mathrm{t}}^{2}+\Delta \mathrm{t}+\sigma_{\mathrm{t}}^{2}\right)^{1 / 2}},
$$

where $\mu_{t}+\Delta t=\sigma_{t}^{2}+\Delta t=\lambda \cdot(t+\Delta t)$ are the mean and the variance of the number of counts obtained during the longer interval, and $\mu_{\mathrm{t}}=o_{\mathrm{t}}^{2}=\lambda t$ are the same statistics for the distribution of counts obtained during the shorter interval.

Although it was assumed by Creelman that the counting process is stationary, i.e., that $\lambda$ is constant, he also assumed that the memory of the counter is imperfect, i.e., that the number of counts held in the counter hyperbolically decayed as a function of the length of the time interval to be remembered. Thus, on the final count, the pulse source of his model acts as if it were nonstationary Poisson with an intensity function $\lambda(t)$ of the form $\lambda /(1+\mathrm{Kt})$, where $K$ is the memory decay factor. Also, Abel $(1972 a, b)$ observed a definite nonstationarity in the counting process; from her data (for $10<\mathrm{t}<$ $500 \mathrm{msec}$ ), the intensity function appears to be something like a negative exponential converging to some nonzero asymptote. Unfortunately, both Creelman and Abel assumed that the intensity functions $\lambda(t)$ and $\lambda(t+\Delta t)$ would be essentially identical for any $t$, that is, that the change in the intensity function between times $t$ and $t+\Delta t$ would be negligible. This assumption is countered by our results, especially by those obtained in Experiments 1,2 , and 4, in which the time difference thresholds $\Delta \mathrm{t}_{\mathrm{d}^{\prime}}=1.0$ were often as large as $25 \%-75 \%$ of the base interval $t$. This is why, for practical reasons, we chose to amend
Creelman's model and made the pulse source's intensity an explicit function of time, in the form of

$$
\lambda(\tau)=b e^{-c t}+\lambda_{0}, \text { for } \tau=t
$$

and

$$
\lambda(\tau)=b e^{-c(t+\Delta t)}+\lambda_{0}, \text { for } \tau=t+\Delta t,
$$

where $b, c$, and $\lambda_{0}$ are constants having the dimension of counts/sec; they are dependent upon the task and individual differences, but not upon the parameters of the time marking stimulus. One should note that such nonstationary neural pulse-source intensity functions are compatible with a class of neurophysiological observations (e.g., Molnar \& Pfeiffer, 1968 , for single cells in the cochlear nucleus).

The turn-on-turn-off stage. In a time discrimination model, the role of the auditory system can be represented by including a stage that precedes the timing stage. This peripheral stage should assume the responsibility of turning the counter mechanism on and off; its simplest form could consist of an energy detector and a threshold device. The acoustic signal passes through the energy detector; whenever the integrated acoustic power reaches the preset threshold, a pulse will be emitted to indicate to the counter the beginning or the end of a physical period to be timed. ${ }^{5}$ The latency, $T$, of the occurrence of this on- and offset pulse, with respect to the on- or offset of the acoustic marker signal, will be a random variable because of noise present in the receptor. Assuming that the internal noise is negligible, this random latency will have a mean, $\mu_{\mathrm{T}}$, and a variance, $\sigma_{\mathrm{T}}^{2}$, inversely proportional to the ratio of the power of the time marker signal and the external noise.

There will be an immediate consequence of including such a sensory-related latency into the model. Predictions for the discriminability of two given intervals will have to be modified, inasmuch as the statistics from which the discriminability index $d^{\prime}$ was computed in Equation 1 will no longer derive from the distribution of the random counts in the timer process alone. Rather, these statistics will have to come from the joint distribution of two independent random variables, namely, the counts in the timer and the latency of the turn-on-turn-off signals. The addition of the random latencies will not change the numerator on the right side of Equation 1, for the means of the latencies associated with turning on and turning off the counter will be the same for the interval of duration $t$ and that of duration $t+\Delta t$. While the mean of the latency distribution will not be represented in Equation 1 because of cancellation, this is not the case for the variance. Actually, the variance sum in the denominator will 
be increased by an extra term, $4 \lambda^{2} \sigma_{T}^{2}$, corresponding to the random latencies present at the beginning and the end of each of the two stimulus intervals-four terms altogether, assuming that all these latencies possess a common distribution.

When building his model, Creelman recognized the need for this added variance term. Since he observed that his variance was larger for low-audibility markers and smaller for salient ones, he regarded the added variance, which he called $\sigma_{v}^{2}$, as a direct effect of random fluctuations in the power of the time marker signal. He found that $\sigma_{\mathrm{v}}^{2}$ can be reasonably well approximated by the empirical expression $A e^{-b v}$, where $A$ and $b$ are constants and $v$ is the signal voltage (the background noise level in his experiments being constant). Thus, Creelman's form of Equation 1 reads as

$$
\mathrm{d}^{\prime} 2 \mathrm{AFC}=\left(2 \frac{\lambda}{1+\mathrm{Kt}} \frac{\Delta \mathrm{t}^{2}}{2 \mathrm{t}+\Delta \mathrm{t}+\sigma_{\mathrm{v}}^{2}}\right)^{1 / 2}
$$

where $t$ and $t+\Delta t$ are the two durations to be discriminated, $\lambda$ is the average firing rate of a stationary Poisson source, $K$ is the decay rate of the counter's memory, and $\sigma_{\mathrm{v}}^{2}$ is the added variance term.

The problem with Creelman's added variance term, $\sigma_{v}^{2}$, however, lies only partly in the empiricism of its definition. The major flaw of this term is that it does not show how the purely physical fluctuations in the power of the time marker acoustic signal will affect time discrimination, that is, a process which is purely perceptual and which operates in the time domain. In other words, there are two links missing in Creelman's $\sigma_{v}^{2}$, both of them important from the conceptual point of view: (1) one that shows the transduction from the physical to the sensory stage, and (2) one that maps this added variability onto the dimension of time. It is for this twofold reason that we chose to propose a second modification to Creelman's model. In our version of the model, we substituted a term signifying fluctuations in the perceptual latency, $T$, for the term depicting fluctuations in the acoustic power. Expressed in such a way, the added variance can reflect the random activity of the neural transducer for acoustic signals, as well as energy fluctuations in these signals, in case they do contain noise.

There are several ways of molding such latency fluctuations into the model. For the sake of conceptual (if not mathematical) simplicity, we chose McGill's (1967) neural counting model for energy detection as our starting point. We did this because, for all practical purposes, the moment of onset or offset of the counting process by an auditory signal should be equivalent to the moment of its detection per se. Therefore, we postulate that detection of a signal occurs when the total amount of neural activity driven by the acoustic input reaches a certain fixed number of discharges, $k$. The latency needed to reach this threshold will certainly have a fixed component reflecting an intangible delay due to the propagation along the length of the neural path extending from the periphery to the timing mechanism, but it will also have a random component, $T_{k}$. The mean and the variance of the distribution of $T_{k}$ has been derived from McGill's model in the Appendix. As shown there, they can be expressed as

$$
\mu_{T_{k}}=k / \alpha P
$$

and

$$
\sigma_{\mathrm{T}_{\mathrm{k}}}^{2}=\mathrm{k} /(\alpha \mathrm{P})^{2}
$$

for nonrandom, deterministic signals, where $\mathrm{k}$ is the threshold number of counts which the auditory neural activity driven by the acoustic signal has to reach before sending a start-stop signal to the counter, $\alpha$ is a matching coefficient (counts/sec) representing the sensitivity of the auditory-neural transducer (it is both a rate factor and a logarithmic transduction factor), and $P$ is the sensation level of the marker (in decibels). However, for noise or noisy signals with a $\mathrm{W}-\mathrm{Hz}$ bandwidth, Equations 4 and 5 must be replaced by the expressions

$$
\mu_{T_{k}}=\tilde{E}\left[T_{k}\right]
$$

and

$$
\sigma_{T_{k}}^{2}=\widetilde{E}\left(T_{k}\right)-\left[\widetilde{E}\left(T_{k}\right)\right]^{2},
$$

where the expectations $\widetilde{E}\left[T_{k}\right]$ and $\widetilde{E}\left[T_{k}^{2}\right]$ are the approximations of the first two moments of the latency distribution as defined by Equations $15 \mathrm{a}$ and $16 \mathrm{a}$ in the Appendix. Thus, our version of Creelman's time discrimination equation, i.e., Equation 3, can be written as

$$
\begin{aligned}
& \mathrm{d}^{\prime}{ }_{2 \mathrm{AFC}} \\
& =\frac{\sqrt{2}[\mathrm{w}(\mathrm{t}+\Delta \mathrm{t})-\mathrm{w}(\mathrm{t})]}{\left\{\mathrm{w}(\mathrm{t})+\mathrm{w}(\mathrm{t}+\Delta \mathrm{t})+2 \sigma_{\mathrm{T}_{\mathrm{k}}}^{2}\left[\lambda^{2}(\mathrm{t})+\lambda^{2}(\mathrm{t}+\Delta \mathrm{t})\right]\right\}^{1 / 2}},
\end{aligned}
$$

where d' $2 A F C$ is the index of discriminability for the time difference $\Delta t$ given the base interval $t ; \lambda(t)$, $\lambda(t+\Delta t)$ are the nonstationary intensity functions of the counting Poisson process, defined in Equations 2 and $2 a$ (counts/sec); $w(t), w(t+\Delta t)$ are

$$
\int_{0}^{t} \lambda(\tau) d \tau \text { and } \int_{0}^{t+\Delta t} \lambda(\tau) d \tau
$$

i.e., the expected number of counts accumulated 
during intervals $t$ and $t+\Delta t$, respectively; and $\sigma_{T k}^{2}$ is the variance of the latency $T_{k}$, i.e., the latency of the onset and offset of the counting process with respect to the onset of the marker stimulus (in $\sec ^{2}-$ note that there are, altogether, four variance terms in the equation since there are four markers that define the two intervals to be compared by the listeners).

'Yo would like to stress that Equation 6 is only $a^{n}$ apnroximation, albeit a very close one, to the exact adaptation of Equation 1 to the present model. Actually, the mean and the variance of the number of counts are conditional mean and variance, since the start/stop latencies are also random variables. In order to bypass additional calculations which would have been unncessarily burdensome, the conditional expectations of the number of discharges emitted by the counter have been computed by using latency expectations. The resulting error has been found to be negligible.

A block diagram of Creelman's model with the present modifications added is presented in Figure 12.

The model and the present data. Using an iterative procedure, values for the parameters $b, c, \lambda_{0}$ (see Equations 2 and $2 a$ ), and $\sigma_{T_{k}}^{2}$ were calculated such that the obtained values minimize the least square error of the resulting discrimination function given in Equation 6, with respect to the complete set of data of a given subject in a given experiment. The obtained parameter values are listed in Table 2 . In the same table there is also a display of the calculated number of neural counts accumulated in the counter during a $25-$, an $80-$, and a $320-\mathrm{msec}$ interval, for the results of our four subjects as well as for the average data of Abel's (1972b) and Creelman's (1962) observers. The reader will note that these numbers calculated for our observers from the data of Experiments 1 and 3 and from our model are somewhat larger, but of the same order of magnitude, as those calculated for Creelman's subjects from his data and from his model. ${ }^{6}$ On the other hand, the variability of the perceptual latency calculated for any of our observers is larger than the figure calculated by Creelman. It is also immediately apparent that both the neural parameters and the accumulated number of counts during any given interval calculated for Abel's data are quite smaller than those that we have found for our observers. This latter discrepancy may reflect the fact that Abel's observers demonstrated a performance inferior to that of either Creelman's or our subjects, probably due to the highstimulus-uncertainty paradigm that Abel used in her experiments.

According to Equation 5, the variability of the counting process's onset-offset latency is inversely related to the marker's power. Thus, Equation 5 and the parameter values found for the two observers in the 65-dB SL condition of Experiment 1 made it possible for us to predict the effect of marker intensity for the same two observers. These predictions are illustrated as the broken lines in Figures $2 a$ and $2 b$. In order to predict the effect of noise marker bandwidth on time discriminability (Experiment 5), we used Equations 15a and 16a in the Appendix, according to which the onset-offset latency of the counting process has a variability also inversely related to the bandwidth of the markers when the markers consist of bursts of Gaussian noise. This predicted effect is illustrated as the broken line in Figure 9.

We would like to point out that although the trends exhibited by the theory and the actual data are essentially identical, the precise time discrimination threshold estimates of the model are, in some cases, quite discrepant from the observed threshold values. There may be several possible reasons for

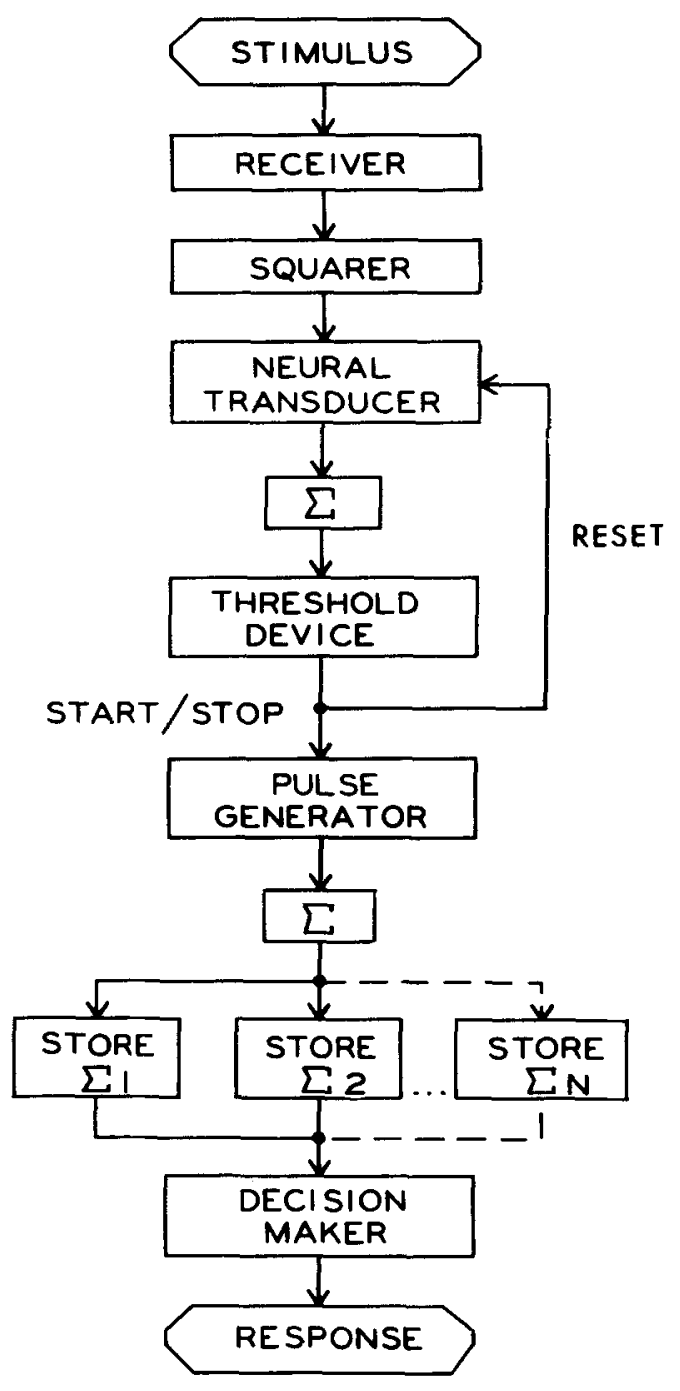

Figure 12. Block diagram of the model. 
Table 2

Model Parameter Values Calculated From Time Discrimination Data

\begin{tabular}{|c|c|c|c|c|c|c|c|c|c|}
\hline & \multirow[b]{2}{*}{ Subject } & \multicolumn{3}{|c|}{$\begin{array}{c}\text { Parameter } \\
\text { (counts/sec) }\end{array}$} & \multicolumn{2}{|c|}{$\begin{array}{l}\text { Standard Deviation } \\
\text { of Perceptual Latency } \\
\left(\sigma_{\mathrm{T}_{k}}^{2}\right)^{1 / 2} \quad \text { (in msec) }\end{array}$} & \multicolumn{3}{|c|}{$\begin{array}{c}\text { Accumulated Number of } \\
\text { Counts During Interval } t^{*} \\
{\left[\int^{t} \lambda(\tau) \mathrm{d} \tau\right]}\end{array}$} \\
\hline & & $\alpha$ & c & $\lambda_{\mathbf{o}}$ & $65 \mathrm{~dB} \mathrm{SL}$ & $5 \mathrm{~dB} \mathrm{SL}$ & $\begin{array}{r}\mathrm{t}=25 \\
\mathrm{msec}\end{array}$ & $\begin{array}{r}\mathrm{t}=80 \\
\mathrm{msec}\end{array}$ & $\begin{array}{c}t=320 \\
\text { msec }\end{array}$ \\
\hline Experiment 1 & $\begin{array}{l}\text { S1 } \\
\text { S2 }\end{array}$ & $\begin{array}{l}41000 \\
13000\end{array}$ & $\begin{array}{l}29.00 \\
11.00\end{array}$ & $\begin{array}{l}2200 \\
1100\end{array}$ & $\begin{array}{r}1.18 \\
.90\end{array}$ & $\begin{array}{l}15.3 \\
11.7\end{array}$ & $\begin{array}{l}784 \\
312\end{array}$ & $\begin{array}{r}1451 \\
780\end{array}$ & $\begin{array}{l}2118 \\
1499\end{array}$ \\
\hline $\begin{array}{l}\text { Creelman (1962) } \\
\text { Abel (1972b) }\end{array}$ & & $300 \dagger$ & $10+$ & $80 \dagger$ & $.04 * *$ & $.152 * *$ & $\begin{array}{r}130 \\
9\end{array}$ & $\begin{array}{r}351 \\
23\end{array}$ & $\begin{array}{r}899 \\
54\end{array}$ \\
\hline
\end{tabular}

*For the present data, $\lambda(\tau)$ is given by Equation 2. For Creelman's $(1962)$ average data, $\lambda(\tau)=\lambda_{a v} /\left(1+K_{a v} \tau\right)$, where $\lambda_{a v}=5,700$ and $K_{a v}=8.11$, as given in Table 2 of his paper.

${ }^{* *} \sigma_{T_{k}}^{2}$ was taken as $\sigma_{v}^{2} / 4 \lambda$, where $\sigma_{v}^{2}$ is Creelman's added variance term that originates in the input's noisiness. The sensation level in the present experiments has to be translated as signal-to-noise ratio in Creelman's experiments, that is, the signal level above masked threshold. These threshold levels were estimated and the variance terms calculated through extrapolation from the intensity parameters given by Creelman.

tApproximated values found in Abel's (1972b) Figure 2.

such a discrepancy, the first of which being that the rigidity of our basic assumptions and the ruggedness of the test may have taken their toll. However, since it appears that the subjects' performance is often superior to that of the model, it cannot be excluded that the subjects could have attended to cues other than strictly time interval cues (such as rhythmic pattern, amount of temporal masking, pitch quality, etc.). It is also likely that the model has been partially defeated by the feature which was supposed to be its chief quality, namely, its simplicity. Later refinements could certainly improve the model's predictive accuracy, but they may also render it less accessible.

Finally, the latency variance of the perceptual response to the onset of a time marker, as given in Equations 5 and $5 \mathrm{a}$, makes it possible to extract numerical values for two "auditory neural" parameters: the sensitivity of the auditory centers that send signals to the internal timer, and the threshold number of discharges that has to be reached before the timing mechanism is actually triggered. Average values of these two parameters were computed for the two subjects participating in Experiment 5. This was accomplished by establishing, from the latency variance estimated for the tone burst marker conditions (i.e., deterministic markers), the set of possible combinations of $\alpha$ and $\mathbf{k}$ satisfying Equation 5. With these combinations, in turn, we went to a table (a larger version of Table 3) which listed the latency variance for a number of bandwidths and parameters $\alpha$ and $k$. We looked up in this table the variances corresponding to the $\alpha-\mathrm{k}$ combinations that we were considering, for a narrow $(350-\mathrm{Hz})$ bandwidth. Among these variance values given by the model, we selected the one which lay closest to the variance estimated from the actual data obtained in the
350-Hz noise marker bandwidth condition. The sensitivity constant, $\alpha$, was found to have a value of 28.0, signifying that the average number of neural discharges evoked by a $65-\mathrm{dB}$ SL signal should be $28 \times 65=1,820 /$ sec. The threshold value, $k$, was found to be 1 . While the absolute magnitude of these numbers has little meaning, the three orders of magnitude difference between threshold and discharge rate is not without interest: it signifies that even a very small acoustically evoked neural activity should be sufficient to trigger off a more central, perceptual-neural response, such as the commencement or termination of the timing process. Since the principal role of the auditory system is to make us aware of changes in our acoustic environment, the model is likely to present a plausible picture of the actual state of affairs.

In addition to making it possible to estimate the variance of the perceptual response latency (= detection latency), our model also permits estimation of the mean of the random component of the latency of auditory detection (Equation 4).

Table 3

Mean and Variance of the Latency Distribution $F_{T_{k}}(t)$ for $65-\mathrm{dB}$ SL Signals

\begin{tabular}{cccrr}
\hline $\begin{array}{c}\text { Bandwidth } \\
(\mathrm{Hz})\end{array}$ & $\begin{array}{c}\alpha \\
\left(\mathrm{sec}^{-1}\right)\end{array}$ & $\mathbf{k}$ & $\begin{array}{r}\mathrm{E}\left[\mathrm{T}_{\mathbf{k}}\right] \\
(\mathrm{msec})\end{array}$ & $\begin{array}{r}\operatorname{Var}\left[\mathrm{T}_{\mathbf{k}}\right] \\
\left(\mathrm{msec}^{2}\right)\end{array}$ \\
\hline \multirow{2}{*}{350} & 10 & 1 & 2.72 & 7.40 \\
& & 6 & 10.63 & 39.23 \\
& 20 & 1 & 1.84 & 3.39 \\
& & 6 & 6.01 & 16.44 \\
1000 & 10 & 1 & 1.99 & 3.92 \\
& & 6 & 9.36 & 20.49 \\
& 20 & 1 & 1.20 & 1.44 \\
& & 6 & 5.07 & 7.70 \\
\hline
\end{tabular}


Similarly to the latency variance, this mean latency, too, is inversely related to the intensity of the auditory marker. In another paper (Divenyi, 1976), it has been shown that the intensity dependence on the mean latency of the auditory detection is almost identical to the intensity dependence exhibited by one of the early components of the auditory evoked potential (Jewett-V or $\left.\mathrm{J}_{\mathrm{V}}\right)^{7}$

\section{CONCLUSION}

The above five experiments were conducted with the specific aim of examining whether or not physical parameters of two successive acoustic markers, that is, their intensity and spectrum, influenced the discriminability of a time interval enclosed between them.

Results of the experiments showed that, at longer intervals ( $80-320 \mathrm{msec})$, temporal discrimination performance was little affected even by large changes in the acoustic properties of markers. Discriminability of shorter intervals $(25 \mathrm{msec})$ exhibited an appreciable dependence on marker properties only in two cases: (1) when the sensation level of one or both markers became very low, and (2) when the two markers were very dissimilar from each other in their spectral composition. These two classes of timeintensity and time-frequency interactions are of definite interest because they reveal certain characteristics of auditory signal processing which may be extremely relevant in speech perception. On the other hand, for all those situations in which the time markers are identical and clearly audible sounds, auditory signals of various intensities and spectra can carry macrotemporal information with equal efficiency.

Finally, the discrimination of brief unfilled intervals may be approximated by Weber's law and can be adequately described by a simple counter model with minor modifications.

\section{APPENDIX \\ FLUCTUATIONS IN THE LATENCY OF THE PERCEPTUAL RESPONSE TO THE ONSET OF AN ACOUSTIC SIGNAL}

We shall attempt to derive here an approximate analytical expression for $\sigma_{T_{k}}^{2}$, the variance of the random variable $T_{k}$ that represents the latency associated with the onset and offset latency of the counting process. Since this latency should be equivalent to the latency of the marker's detection, it appeared logical for us to base our derivation on an energy detector model similar to the one proposed by McGill (1967). McGill's model assumes that a signal, $x(t)$, is detected as the cumulative output, $N(t)$, of a neural, Poisson transducer with expected pulse output $\alpha y(t)$, where $\alpha$ is a constant and $y(t)=\int_{0}^{t} x^{2}(\tau) d \tau$ is the energy of the input signal. In our present model, we shall postulate a threshold device which, whenever $N(t)$ reaches a certain number $k$, resets the process $N(t)$ to zero and simultaneously sends a "start" or "stop" signal to the internal timer. Thus, we seek the variance $\sigma_{T_{k}}^{2}$ of the waiting time $T_{k}$ for the process $N(t)$ to reach the barrier $k$, where our new random variable $T_{k}$ is defined as $\inf \{t: N(t)=k\}$, $0<\mathrm{t}<\infty, \mathrm{k}=1,2, \ldots$

Intuitively, we would expect $\sigma_{\mathrm{T}_{\mathrm{k}}}^{2}$ to be monotonically related to the threshold $\mathrm{k}$ and inversely related to the transducer output rate and to the time-average power of the input signal $x(t)$. If the acoustic input contains noise, i.e., if it has its own variance, we would expect $\sigma_{\mathrm{T}_{k}}^{2}$ to be a monotonic function of the amount of fluctuations in the power of $x(t)$. Our aim is to derive at least an acceptable approximation to the variance term $\sigma_{T_{k}}^{2}$ while having recourse to a minimum number of assumptions.

Because the process $N(t)$ is strictly nondecreasing in time (i.e., a pure birth process).

$$
P\left[T_{k}<t\right]=P[N(t) \geqslant k] .
$$

Therefore, if $\mathrm{p}_{\mathrm{N}(\mathrm{t})}(\mathrm{k})$ is the probability that at time $\mathrm{t}$ the value of the process $N(t)$ is exactly $k$, the distribution of $T_{k}$ is given as

$$
F_{T_{k}}(t)=\sum_{j=k}^{\infty} p_{N(t)}(j)=1-\sum_{j=0}^{k-1} p_{N(t)}(j),
$$

with the associated probability density

$$
f_{T_{k}}(t)=-\frac{\partial}{\partial t} \sum_{j=0}^{k-1} p_{N(t)}(j)
$$

The first two moments of this density are given by

$$
E\left[T_{k}\right]=-\sum_{j=0}^{k} \int_{0}^{\infty} t \frac{\partial}{\partial t} p_{N(t)}(j) d t
$$

and

$$
E\left[T_{k}^{2}\right]=-\sum_{j=0}^{k} \int_{0}^{\infty} t^{2} \frac{\partial}{\partial t} p_{N(t)}(j) d t .
$$

Whenever the input process $x(t)$ is deterministic, $N(t)$ will be a simple Poisson process with $p_{N}(t)(j)=\exp [-\alpha y(t)]$ $[\alpha y(t)]^{j} / j$ !. In this case, $T_{k}$ will be distributed as a gamma random variable (Parzen, 1960, p. 261), with

$$
\mathrm{E}\left[\mathrm{T}_{\mathrm{k}}\right]=\mathrm{k} / \alpha \mathrm{P}
$$

and

$$
\left.\operatorname{Var}\left[\mathrm{T}_{k}\right]=k / \alpha \mathrm{P}\right)^{2},
$$

where $P$ is the time-average power of the input $x(t)$.

On the other hand, when $x(t) \equiv \bar{X}(t)$ is a stochastic process, e.g., Gaussian noise with bandwidth $W$ and spectral power density $\mathbf{N}_{0}$, finding the derivative indicated in Equations 9 and 10 may prove itself a complicated, if not entirely hopeless, task. Fortunately, there are valid approximations available. For the Gaussian case, we may use the simplified expression for $\bar{Y}(t)$, the energy of $\bar{X}(t)$, that follows from Rice's representation of a bandlimited Gaussian noise process as an ensemble of $\mathrm{Wt}$ sine 
and Wt cosine components (Green \& Swets, 1966, chap. 6). According to this representation, a waveform $\bar{X}(t)$ is uniquely determined by amplitude samples $X_{i}$ taken at regular intervals $t_{i}, i=1,2, \ldots$, at $\Delta t \leqslant 1 / 2 W$ seconds apart. This means that the samples $X_{i}$ will be Gaussian variables with an associated covariance matrix $K$ consisting of the covariance terms $\bar{X}_{\mathbf{i}} X_{\mathbf{i}}$. When the sampling interval $\Delta t$ is exactly $1 / 2 W$, all off-diagonal elements of $K$ vanish and

$$
\bar{Y}(t)=\sum_{i=1}^{2 W t} X_{i}^{2} / 2 W
$$

Although it is true that such a noise representation is highly idealized and that its statistics are different from those pertaining to more realistic, continuous noise representations (Slepian, 1958), the approximation is an acceptable one, especially when $2 \mathrm{Wt}$ is large. Thus, with the constraint that $\Delta \mathrm{t}=1 / 2 \mathrm{~W}$, one may arrive at a significant computational economy when calculating statistics of quantities related to $\bar{Y}(t)$. When using this noise representation, it can be shown (McGill, 1967) that $N(t)$ is distributed as a negative binomial random variable with probability weights

$$
\begin{aligned}
\mathrm{p}_{\mathrm{N}(\mathrm{t})}(\mathrm{j}) & =f(\mathrm{j} ; \mathrm{Wt}, 1-\mathrm{q})=\left(\begin{array}{c}
\mathrm{Wt}+\mathrm{j}-1 \\
\mathrm{j}
\end{array}\right) \mathrm{q}(1-\mathrm{q})^{\mathrm{W} t}, \\
\quad 0<\mathrm{t}<\infty & \\
\mathrm{q} & =\alpha \dot{\mathrm{P}} /(\mathrm{W}+\alpha \mathrm{P}) \\
\mathbf{P} & =\mathrm{WN}_{\mathrm{o}} .
\end{aligned}
$$

However, the derivative $\partial / \partial \operatorname{tp}_{N(t)}(j)$ is meaningless as long as $\mathrm{t}=\mathrm{i} / 2 \mathrm{~W}, \mathrm{i}=1,2, \ldots$, is discrete, unless $\mathrm{W} \rightarrow \infty$, i.e., unless the noise becomes a true white noise-in which case it has infinite power and our endeavor is at a dead end. On the other hand, for any finite bandwidth, the derivative $\partial / \partial \operatorname{tpN}(t)(j)$ exists only when $t$ is continuous-in which case the covariance matrix $K$ will contain nonzero offdiagonal elements, i.e., the samples $X_{i}$ will no longer be independent and, by consequence, calculation of related statistical quantities will become prohibitively complex (Schwartz, 1970). To get out of this impass, we elected to follow the convenient way of ignoring all off-diagonal cells in the matrix $K$, i.e., of considering all samples $X_{i}$ independent. Such an assumption, naturally, violates Rice's condition for the representation of a band-limited Gaussian process, especially when the duration of the noise signal is short. Therefore, $E\left[T_{k}\right]$ and $\operatorname{Var}\left[T_{k}\right]$ computed this way will be distorted approximations of the true mean and variance of the variable $T_{k}$; this is why we shall henceforth label them as $\left.\widetilde{E}_{[} T_{k}\right]$ and $\widetilde{\operatorname{Var}}\left[T_{k}\right]$. Fortunately, it is only the Rice noise representation which is to be violated by our assumption of independence of the noise samples. Continuity of the time parameter does not change the distribution of $T_{k}$ because the negative binomial distribution is infinitely divisible (Feller, 1968, p. 291). Thus, in probability theory at least, the derivative $\partial / \partial \operatorname{tp}_{N(t)}(j)$ exists for all $t>0$ and Equations 9 and 10 are meaningful.

The central moments $\tilde{\mathrm{E}}\left[\mathrm{T}_{k}\right]$ and $\widetilde{\operatorname{Var}}\left[\mathrm{T}_{k}\right]$ can be expressed analytically in several different ways but, regrettably, none of these appears to be truly simple. One such expression is derived from a particular transformation of the tail of a negative binomial distribution (Olkin \& Sobel, 1965):

$$
\begin{aligned}
\mathrm{FWT}_{\mathrm{k}}(\nu) & =1-\sum_{\mathrm{j}=0}^{\mathrm{k}} \mathrm{\sum}_{\mathrm{N}(\nu)}^{1}(\mathrm{j}) \\
& =\mathrm{I}_{\mathrm{q}}(\mathrm{k}, v)=1-\mathrm{I}_{1}-\mathrm{q}(\nu, \mathrm{k})
\end{aligned}
$$

and

$$
\begin{aligned}
\mathrm{f}_{\mathrm{WT}_{\mathrm{k}}(v)} & =\frac{\partial}{\partial v}\left[1-\mathrm{I}_{1-\mathrm{q}}(v, \mathrm{k})\right] \\
& =-\frac{\partial}{\partial v} \mathrm{I}_{1-\mathrm{q}}(v, \mathrm{k}),
\end{aligned}
$$

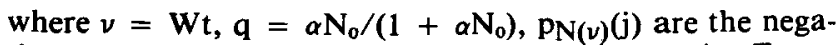
tive binomial probability weights as shown in Equation 12 and $I_{x}(a, b)=[\Gamma(a+b) / \Gamma(a) \Gamma(b)] \int_{0}^{x} s^{a}-1$ $(1-s)^{b-1 d s,} 0<x \leqslant 1$, is Pearson's incomplete beta function (beta distribution). Thus, the first two moments of $F_{T_{k}}(t)$ can be expressed as

$$
\begin{aligned}
\tilde{E}\left[T_{k}\right] & =-W^{-1} \int_{0}^{\infty} \nu \frac{\partial}{\partial v} I_{1-q}(v, \mathbf{k}) d v \\
& =-\left.W^{-1}\left[\nu I_{1}-q(v, \mathbf{k})\right]\right|_{0} ^{\infty}+W^{-1} \int_{0}^{\infty} I_{1-q}(v, k) d v
\end{aligned}
$$

and

$$
\begin{aligned}
\widetilde{\mathrm{E}}\left[\mathrm{T}_{\mathrm{k}}{ }^{2}\right] & =-\mathrm{W}^{-2} \int_{0}^{\infty} \nu^{2} \frac{\partial}{\partial \nu} \mathrm{I}_{1}-\mathrm{q}(v, \mathrm{k}) \mathrm{d} \nu \\
& =-\left.\mathrm{W}^{-2}\left[\nu^{2} \mathrm{I}_{1}-\mathrm{q}(\nu, \mathrm{k})\right]\right|_{0} ^{\infty}+\frac{2}{\mathrm{~W}^{2}} \int_{0}^{\infty} \nu \mathrm{I}_{1-\mathrm{q}}(\nu, \mathrm{k}) \mathrm{d} \nu .
\end{aligned}
$$

Since $I_{1-q}(\nu, k)=O\left(v^{-3}\right)$, the first term in Equations 15a and $16 \mathrm{a}$ vanish. Unfortunately, however, an explicit solution of the remaining integral terms is complicated unless $\mathbf{k}$ is very small. Alternative methods (e.g., use of the Laplace transform, etc.) also fail to appreciably simplify the task. For this reason, a numerical analysis was performed, in order to study the effect of changes in the parameters $\alpha, k, N_{0}$, and $W$ on the mean and the variance of our waiting time distribution. The results of this analysis were used to build a rather extensive table. Table 3 illustrates a much shortened version of the larger table; it shows mean and variance values computed for parameter values $\alpha=\{10,20\}, \mathrm{k}=\{1,6\}$, and $\mathrm{W}=\{350,1000\}$, for $P=W_{0}=65 \mathrm{~dB}$. It is immediately apparent that this table does confirm our predictions: as either $k$ increases, $a$ decreases, or $\mathrm{W}$ decreases, both the mean and the variance of the distribution $\mathrm{F}_{\mathrm{T}_{\mathrm{k}}}(\mathrm{t})$ increase.

An additional point is worth noting. It is known (Feller, 1968 , p. 281) that as $W \rightarrow \infty$. and $N_{0} \rightarrow 0$ such that $\mathrm{P}=\mathrm{WN}_{\mathrm{o}}$ remains constant, the probabilities $f\left(\mathrm{j} ; \mathrm{W}_{\mathrm{t}}\right.$, $\left.1 / 1+\alpha \mathrm{N}_{0}\right) \rightarrow \mathrm{e}^{-\alpha \mathrm{Pt}}(\alpha \mathrm{Pt})^{\mathrm{j}} / \mathrm{j}$ !, i.e., the negative binomial distribution will approach the Poisson distribution. Therefore, as the bandwidth of constant-SPL noise markers 
becomes infinite, that is, as the noise becomes true white noise, fluctuations in the noise power will no longer affect statistics of the waiting times $T_{k}$, which thus will be distributed as gamma random variables with mean and variance identical to those shown in Equations $7 \mathrm{a}$ and $7 \mathrm{~b}$. In other words, as the bandwidth of a Gaussian process whose energy drives a Poisson process approaches infinity, the compound Poisson process will behave as if the driving process were deterministic, i.e., it will become a simple Poisson point process. This point has been proven by the results of Experiment V (Figure 9).

\section{REFERENCES}

ABEL, S. M. Duration discrimination of noise and tone bursts. Journal of the Acoustical Society of America, 1972, 51, 1219-1223. (a)

ABEL, S. M. Discrimination of temporal gaps. Journal of the Acoustical Society of A merica, 1972, 52, 519-524. (b)

Abramson, A. S., \& Lisker, L. Discrimination along the voicing continuum: Cross-language tests. In Proceedings of the Sixth International Congress of Phonetic Sciences, Prague, 1967. Prague: Academia Publishing House of Czechoslovak Academy of Science, 1970. Pp. 569-573.

Allan, L. G., \& Kristofferson, A. B. Psychophysical theories of duration discrimination. Perception \& Psychophysics, 1974, 16, 26-34.

Bregman, A. S., \& Campbell, J. L. Primary auditory stream segregation and perception of order in rapid sequences of tones. Journal of Experimental Psychology, 1971, 89, 244-249.

Chistovich, L. A. Discrimination of the time interval between two short acoustic pulses. Soviet Physics: Acoustics, 1959, 5, 493-496.

Collyer, C. E. The detection of a temporal gap between two disparate stimuli. Perception \& Psychophysics, 1974, 16, 96-100.

Creelman, C. D. Human discrimination of auditory duration. Journal of the Acoustical Society of America, 1962, 34, 582-593.

DANN ER, W. F. Discrimination of short unfilled temporal intervals bounded by nonspeech sounds. Unpublished Master's thesis, Washington University, 1975 (Psychology).

DivenYI, P. L. The rhythmic perception of micro-melodies: Detectability by human observers of a time increment between sinusoidal pulses of two different, successive frequencies. In $\mathrm{E}$. Gordon (Ed.), University of Iowa studies in the psychology of music (Vol. 7). Lowa City: The University of Iowa Press, 1971. Pp. 41-130.

DivenYi, P. L. On the latency of auditory detection. In S. K. Hirsh, D. H. Eldredge, 1. J. Hirsh, \& S. R. Silverman (Eds.), Hearing and Davis, Essays honoring Hallowell Davis. St. Lou is: Washington University Press, 1976. Pp. 225-234.

Divenyi, P. L., \& DANNER, W. F. Non-monotonic discrimination functions for time intervals: Implications for VOT perception. Journal of the Acoustical Society of America, 1975, 58, S36(A).

Divenyi, P. L., DANner, W. F., \& Bieker, J. J. Discrimination of the temporal separation between pairs of sinusoidal bursts. Journal of the Acoustical Society of America, 1974, 55, S31(A).

EFroN, R. Effect of stimulus duration on perceptual onset and offset latencies. Perception \& Psychophysics, 1970, 8, 231-234.

FELlER, W. An introduction to probability theory (Vol. I, 3d ed.). New York: Wiley, 1968.

FRASER, J. T. (Ed.) The voices of time. New York: Braziller, 1966.

GETTY, D. J. Discrimination of short temporal intervals: A comparison of two models. Perception \& Psychophysics, 1975, 18, 1.8.

GrEen, D. M., \& SwETS, J. A. Signal detection theory and psychophysics. New York: Wiley, 1966.
GreenberG, G. Z., \& Larkin, W. D. Frequency-response characteristic of auditory observers detecting signals at a single frequency in noise: The probe-signal method. Joumal of the Acoustical Society of America, 1968, 44, 1513-1523.

HENRY, F. M. Discrimination of the duration of a sound. Journal of Experimental Psychology, 1948, 45, 734-743.

Hirsh, I. J., \& SHerrick, C. Perceived order in different sense modalities. Journal of Experimental Psychology, 1961, 62, 423-432.

KUHL, P., \& Micler, J. D. Speech perception by the chinchilla: The voiced-voiceless distinction in alveolar plosive consonants. Science, 1975, 190, 69-72.

Liberman, A. M., Harris, K. S., Kinney, J. A., \& Lane, H. The discrimination of relative onset time of the components of certain speech patterns. Journal of Experimental Psychology, 1961, 61, 379-388.

McGiLL, W. J. Neural counting mechanisms and energy detection in audition. Journal of Mathematical Psychology, 1967, 4, 351-376.

Miller, J. D., Pastore, R. E., Wier, C. C., Kelly, W. J., \& Dooling, R. J. Discrimination and labeling of noise-buzz sequences with varying noise-lead times: An example of categorical perception. Journal of the Acoustical Society of America, $1976,60,410.417$

Molnar, C. E., \& Pfeiffer, R. R. Interpretation of spontaneous spike discharge patterns of neurons in the cochlear nucleus. Proceedings of the Institute of Electrical \& Electronic Engineers, $1968,56,993-1004$.

Parzen, E. Modern probability theory and its applications. New York: Wiley, 1960.

Olkin, I., \& Sonel, M. Integral expressions for tail probabilities of the multinomial and negative multinomial distributions. Biometrika, 1965, 52, 167-179.

PEnNer, M. J. The perceptual offset: A problem of decision criteria. Perception \& Psychophysics, 1975, 17, 587-590.

SCHWARTz, M. I. Distribution of the time-average power of a Gaussian noise process. Institute of Electrical \& Electronic Engineers Transactions on Information Theory, 1970, IT-16, $17-26$.

Slepian, D. Fluctuations of random noise power. Bell System Technical Journal, 1958, 37, 163-184.

STUDDERT-KenNedy, M. Speech perception. In N. J. Lass (Ed.), Contemporary issues in experimental phonetics. Springfield, III: Thomas, 1975 . .

Treisman, A. M. Contextual cues in selective listening. Quarterly Journal of Experimental Psychology, 1960, 12, 242-248.

VAN NOORDEN, L. P. A. S. Discrimination of time intervals bounded by tones of different frequencies. Instituut voor Perceptie Ornderzook (Eindhoven, The Netherlands) Annual Progress Report, 1971, 6, 12-15.

Williams, K. N., \& Perrotr, D. R. Temporal resolution of tonal pulses. Journal of the Acoustical Society of America, 1972, 51, 644-647.

\section{NOTES}

1. Because of the filter's skirts being displaced on a logarithmic rather than on a linear scale, a nominal $350-\mathrm{Hz}$ bandwidth was actually wider (on a linear frequency scale) when its center frequency was $4 \mathrm{kHz}$ than when its center frequency was $500 \mathrm{~Hz}$. On the other hand, the upper skirt of the $2.8-\mathbf{k H z}$-wide noise centered at $4 \mathrm{kHz}$ was limited by the low-pass nature of the TDH 49 earphones.

2. The reason why Figure 10 shows only one half of the hypothetical filter is that only descending frequency changes were tested in Experiment 4.

3. In the Procedure section, it was mentioned that the intervals in the present study were measured from marker onset to marker onset, rather than from marker offset to marker onset (i.e., the 
gap) as done by Abel (1972b). To compensate for this procedural discrepancy, her data shown in Figure 12 represent a base interval that is 5 msec longer than the original intervals given by her, since the duration of her markers was $5 \mathrm{msec}$.

4. However, Creelman obtained his data using tones at intensities close to their threshold of audibility, i.e., 5 to $20 \mathrm{~dB}$ above masked threshold, as opposed to the 86-dB SPL markers, the results for which are compared with results of other authors in Figure 11. Subsequent computations showed that Creelman's observers performed slightly better than ours with signals presented at $10 \mathrm{~dB}$ SL.
5. It is easy to imagine an electronic device that would respond to signal offsets in a way similar to an integrator responding to signal onsets.

6. We computed Creelman's $\lambda(t)$ values as $\lambda /(1+\mathrm{Kt})$, as given in his model equation.

7. In this comparison of psychophysically derived and electrophysiological latencies, we have disregarded an additive constant which represents a fixed neural propagation delay.

(Received for publication September 15, 1975, revision accepted November $18,1976$. .) 